International Journal of Kurdish Studies

(ISSN:2149-2751)

4 (2), pp. 350-364

http://www.ijoks.com

\title{
The concept of lifestyle: Theoretical Study
}

Abdullah Kurshid Abdullah ${ }^{1}$

Received: Feb 05, 2018
Shahlaa Wali Jabbar ${ }^{2}$

Reviewed: Mar 08, 2018
Accepted: Mar 15, 2018

\begin{abstract}
This paper tries to investigate lifestyle concept, as lifestyle has been a hot topic to research on by sociologists, psychologists, anthropologists and medical experts due to its important role on the social, cultural, psychological and political aspects of life. The different experts have studied the concept in a different lens; this academic effort looks at it sociologically. The concept occurred in the modern era that includes the variables of social classes, options, and individual behaviors, in which led identity creation. Moreover, it also discusses modernity and its era, such as; development, mass production equipment, technology and increasing social, cultural and symbolic capitals. Those interrelated concepts led to have a different lifestyle and identity.
\end{abstract}

Keywords:Lifestyle, Modernity, Distinction, Consumption, Social Class

\section{Recommended citation:}

Abdullah A. K. \& Jabbar S. W. (2018). The concept of lifestyle: Theoretical Study. International Journal of Kurdish Studies 4 (2), 350-364. doi: 10.21600/ ijoks.454453

\footnotetext{
${ }^{1}$ Assc. Prof. Dr., Social Organization, Salahaddin University, College of Art, Sociology, Erbil, Iraq. E-mail: abdullah.abdullah@su.edu.krd

${ }^{2} \mathrm{Ph} . \mathrm{D}$ Student and Lecturer, Urban Sociology, Salahaddin University, College of Art, Sociology, Erbil, Iraq, Email: shahlaa.jabbar@su.edu.krd
} 


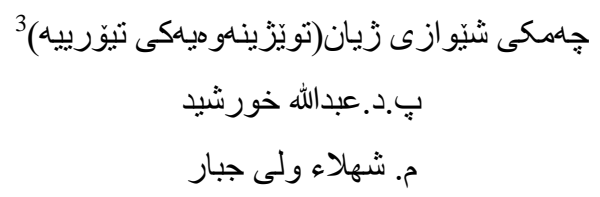

بوخته

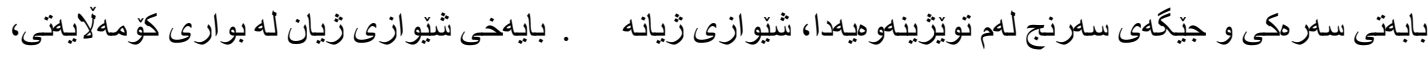

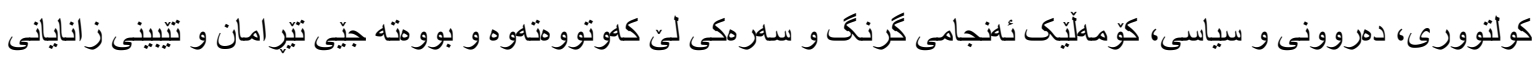

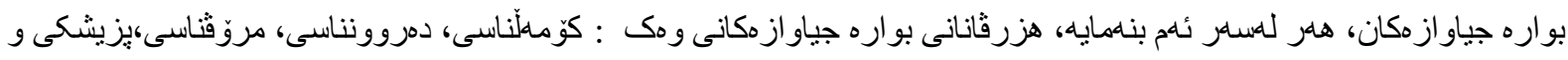

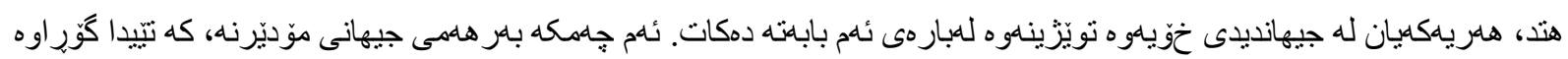

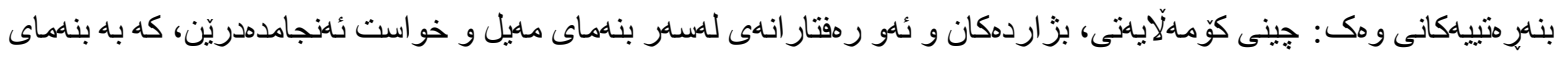

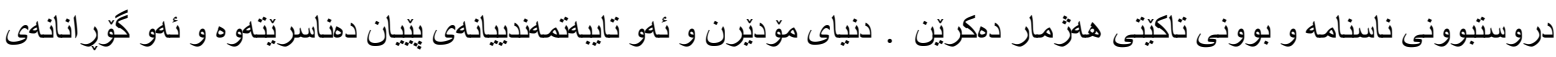

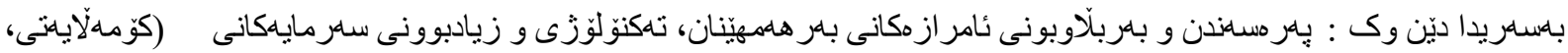

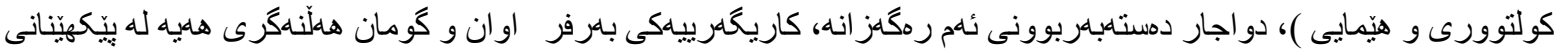

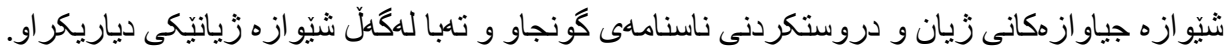

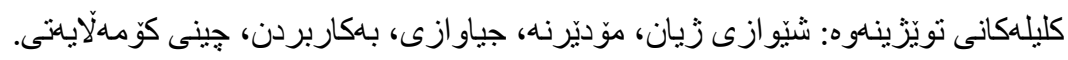

\section{بيَيشْكى}

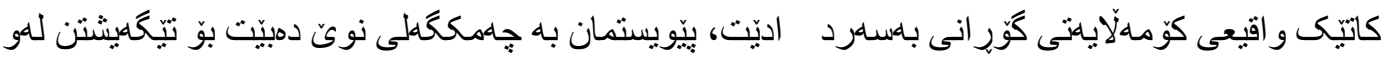

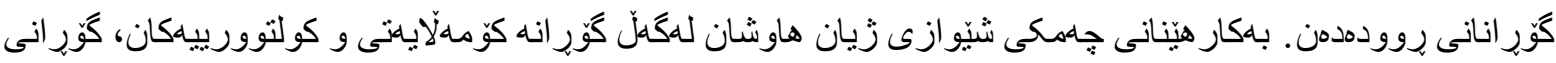

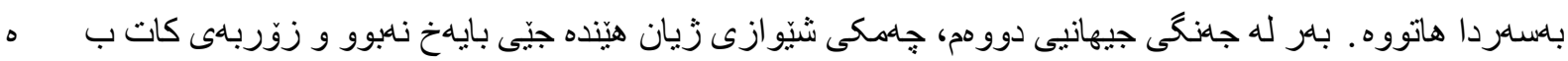

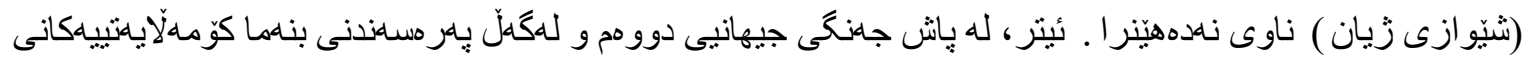

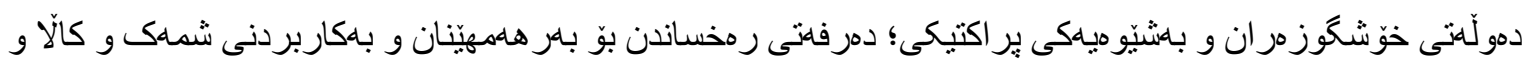

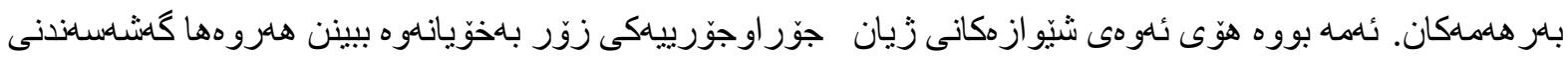

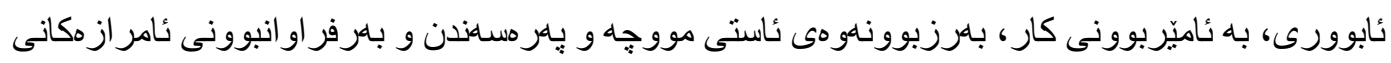

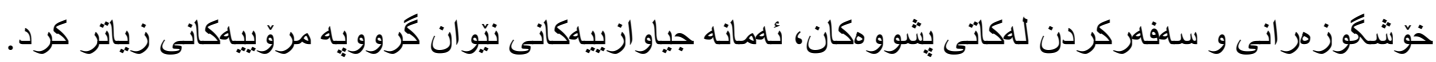

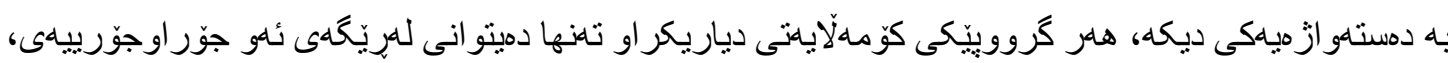

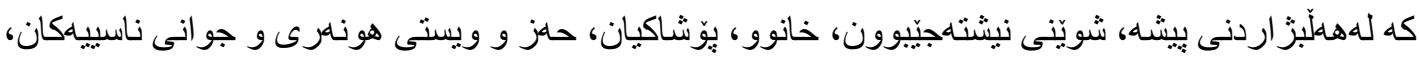

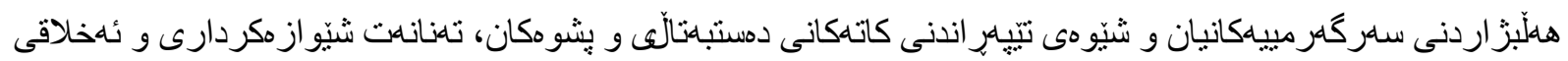

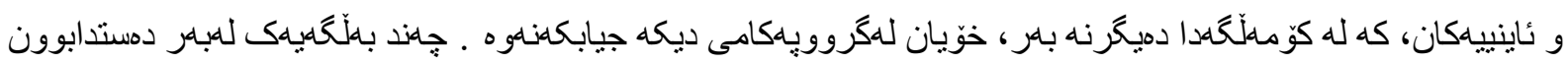

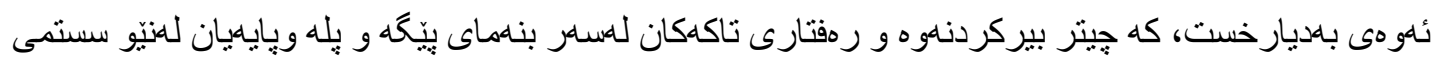

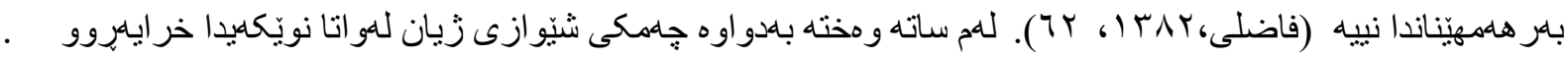

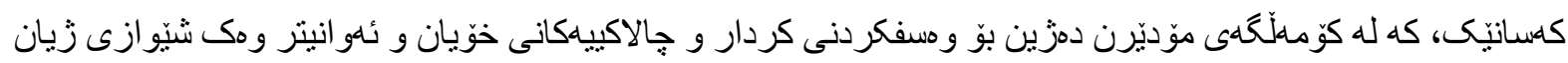

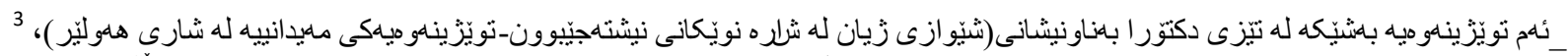

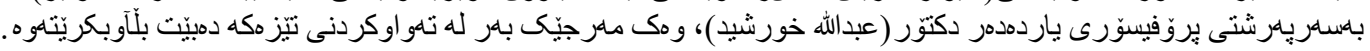




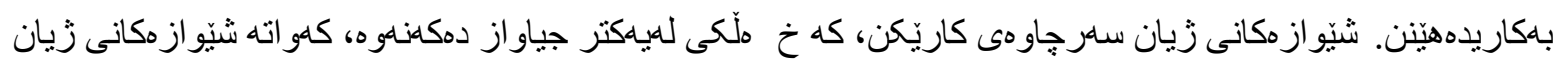

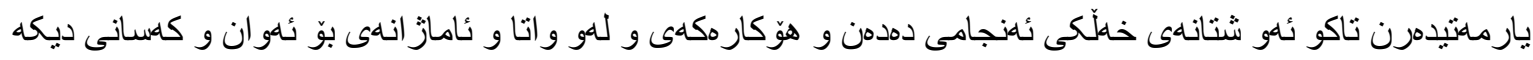

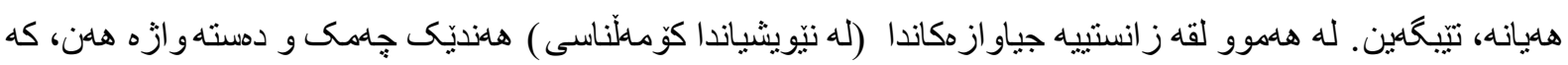

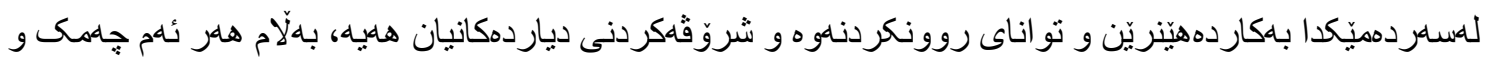

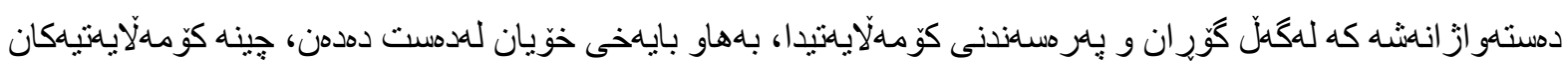

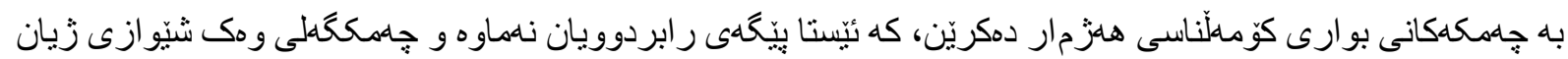
و بهكاربردن بوون به جيّكر هو هيان.

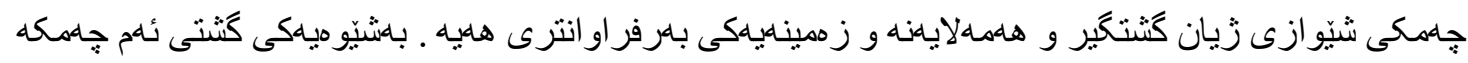

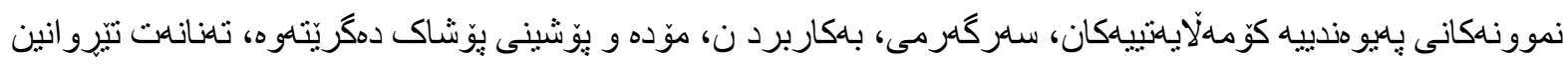

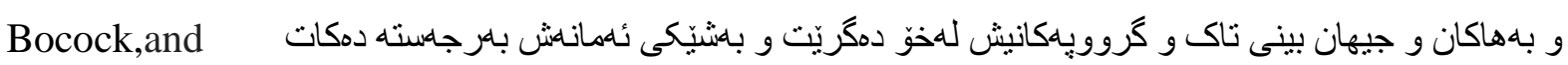

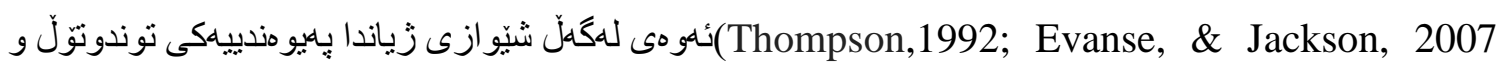

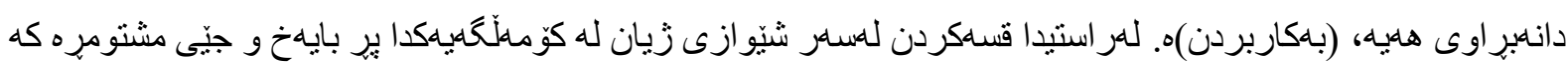

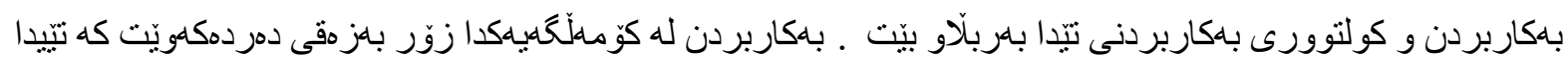

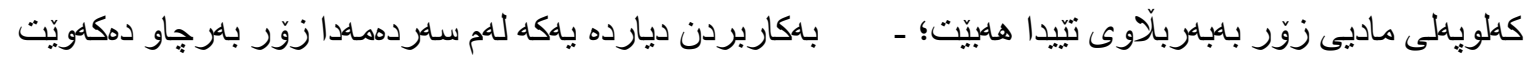

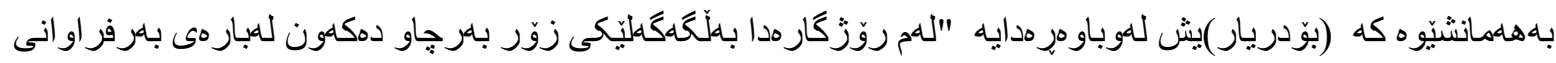

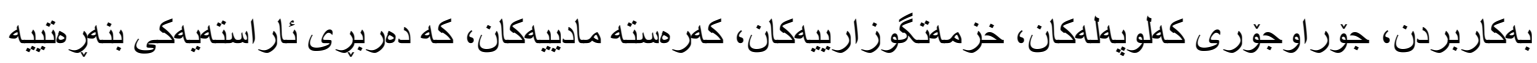

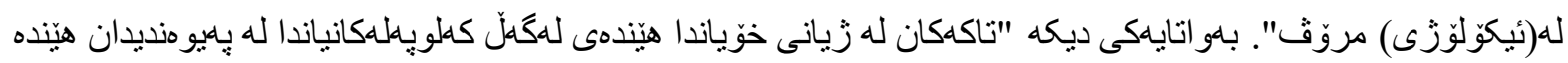

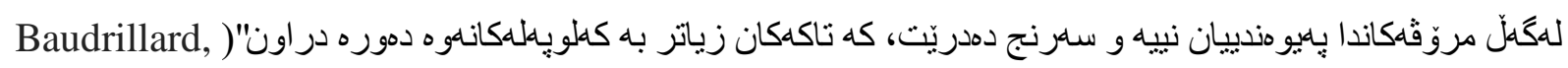

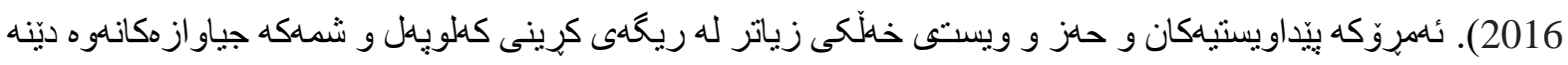

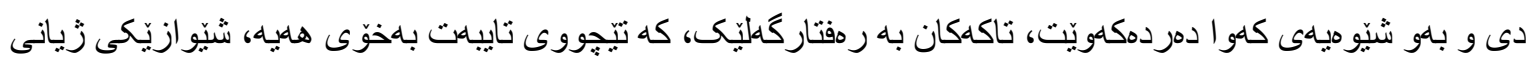
تاييهت دمكرنهبهر.

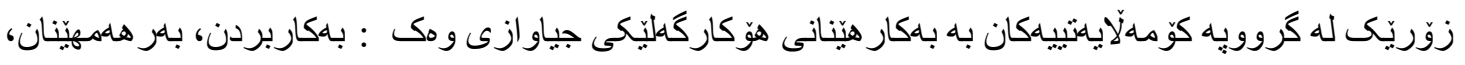

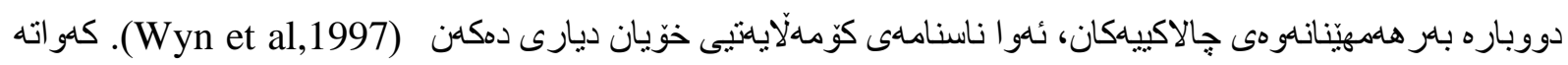

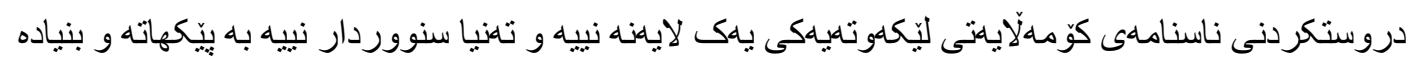

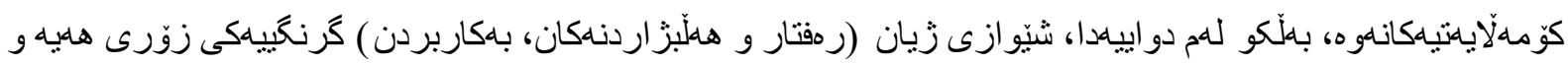

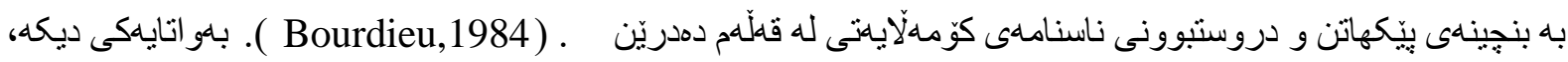

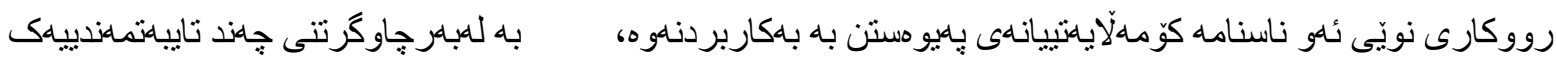

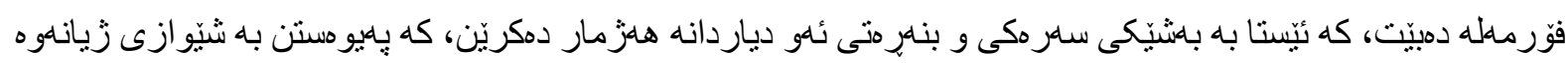

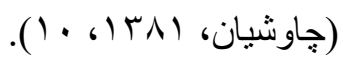

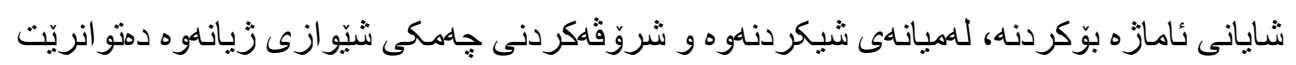

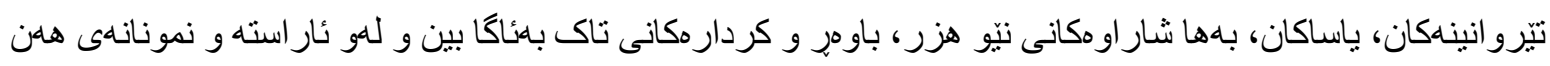

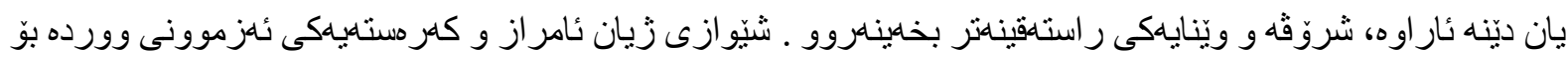

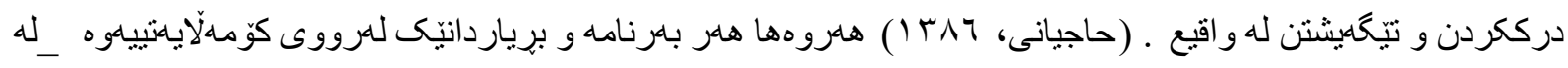

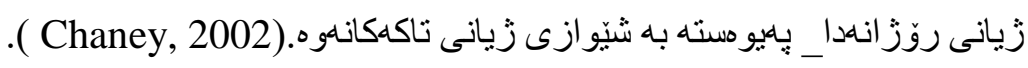




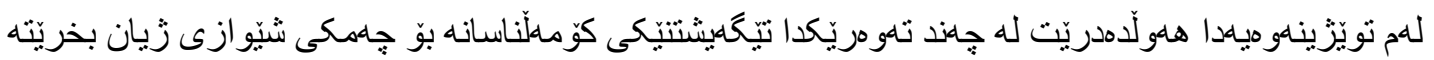

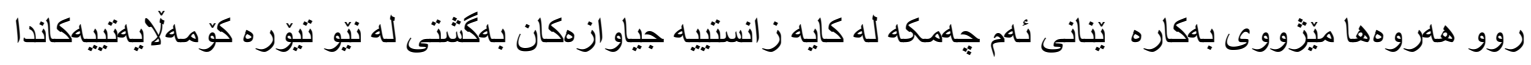

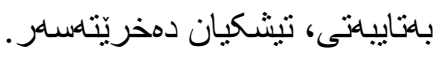

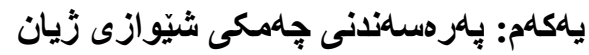

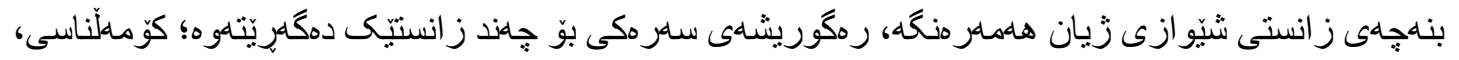

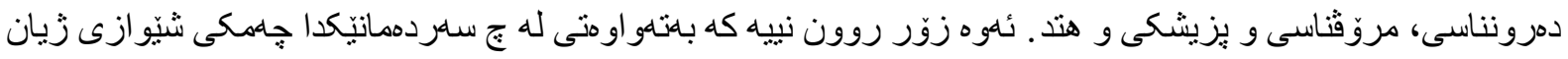

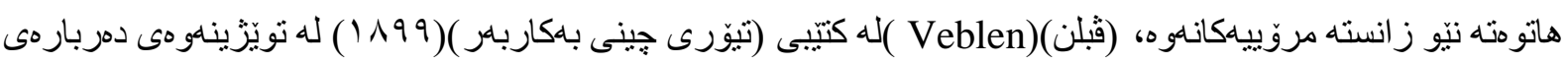

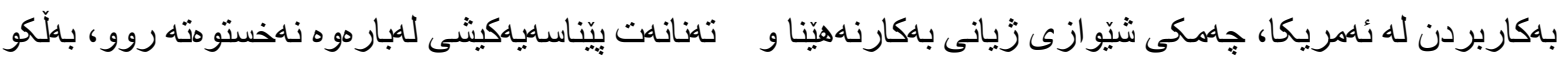

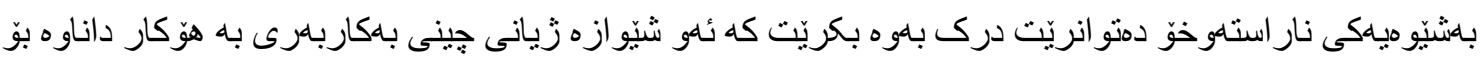

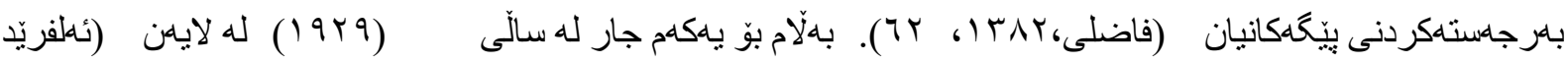

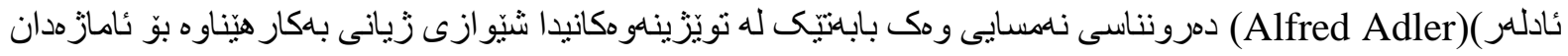

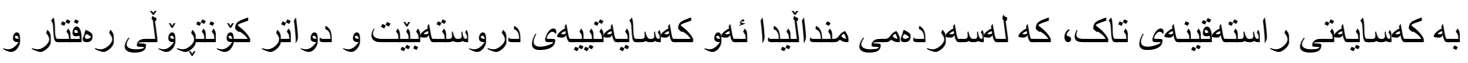

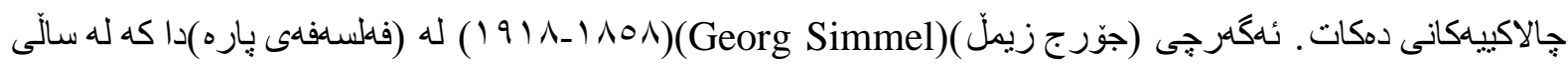

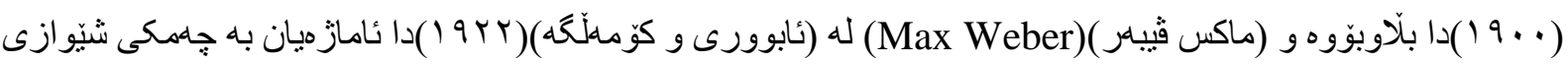

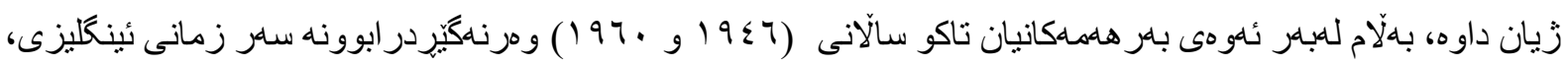

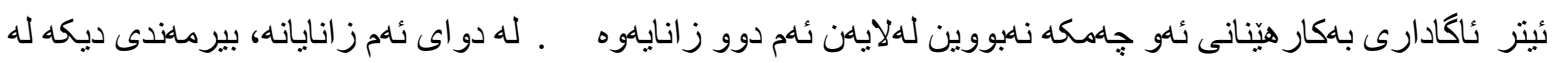

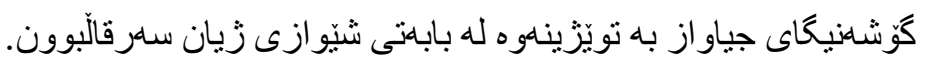

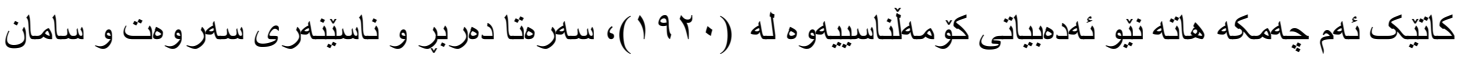

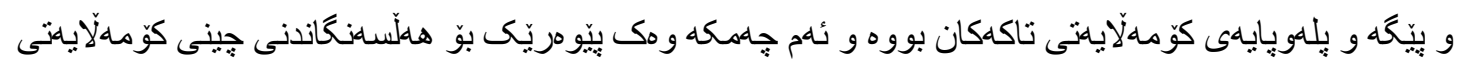

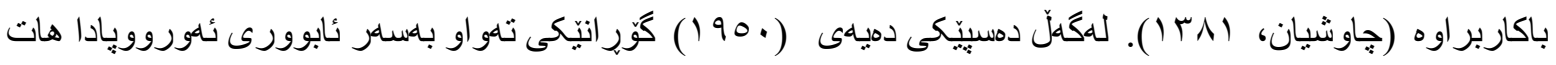

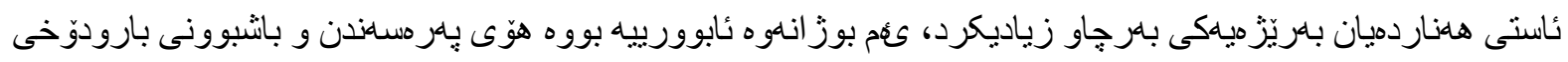

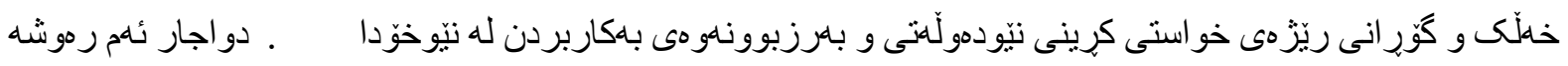

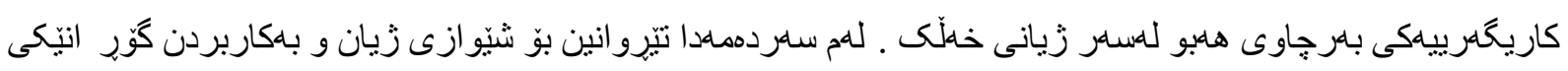

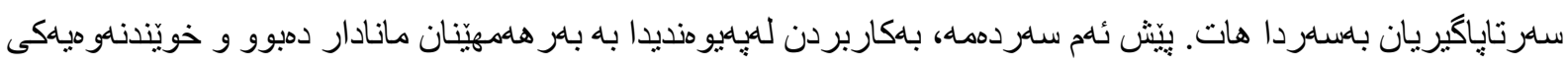

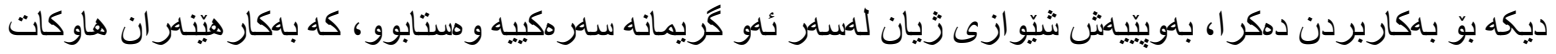

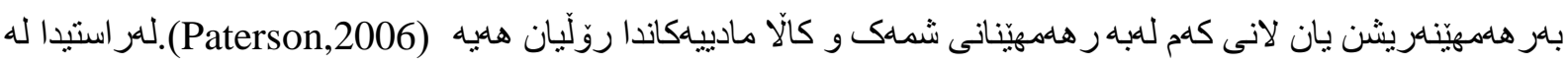

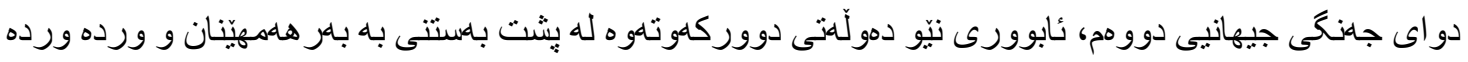

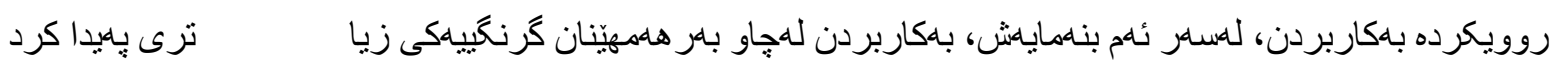

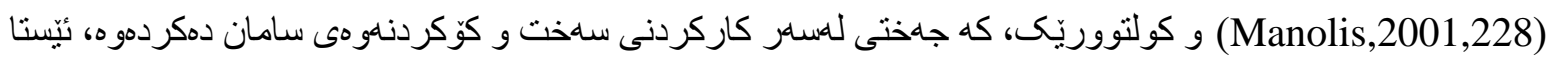

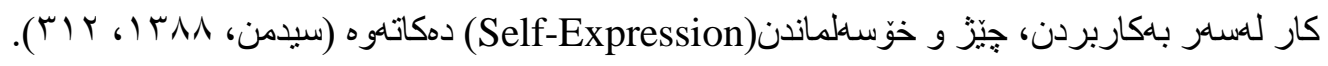

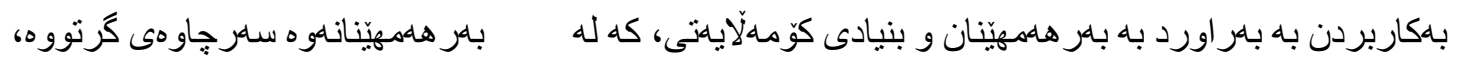

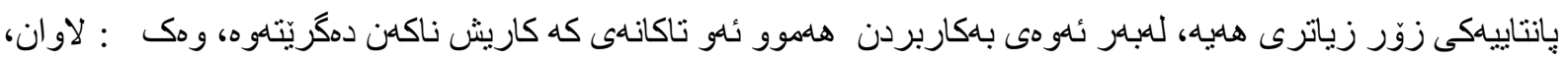




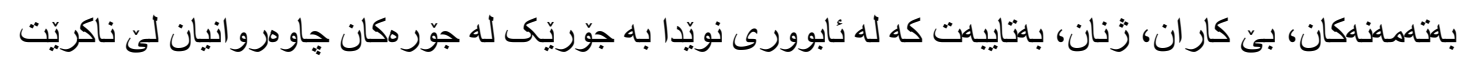

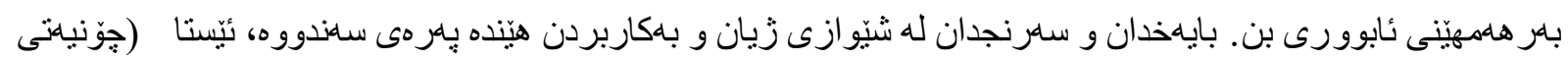

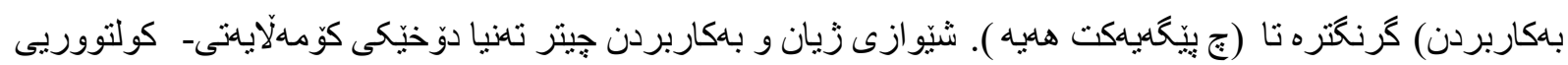

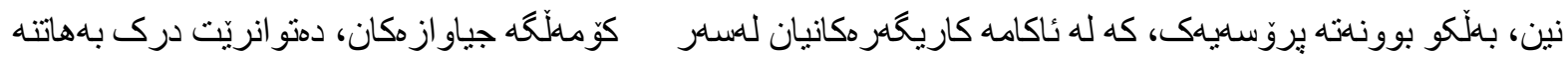

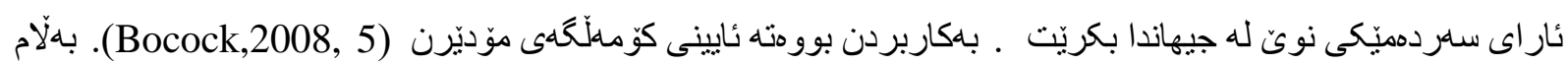

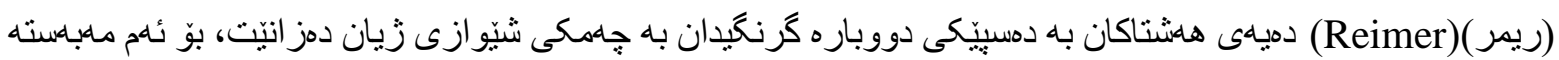

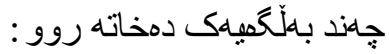

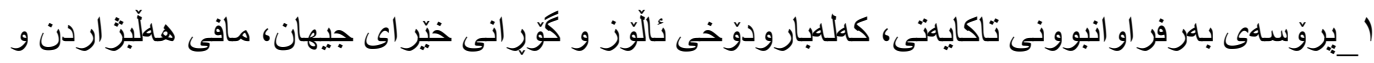

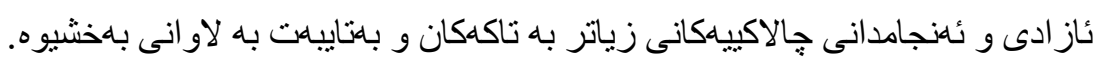

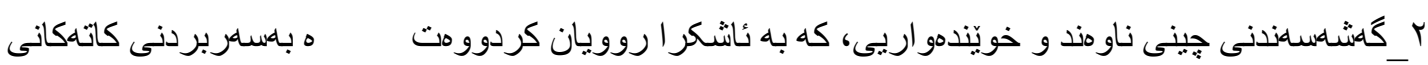

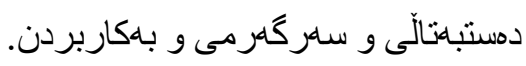

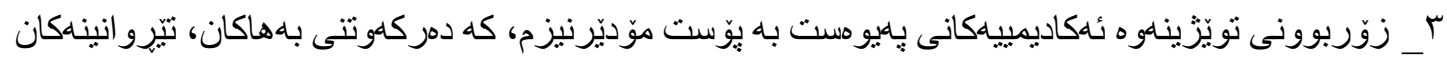

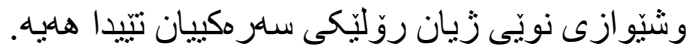

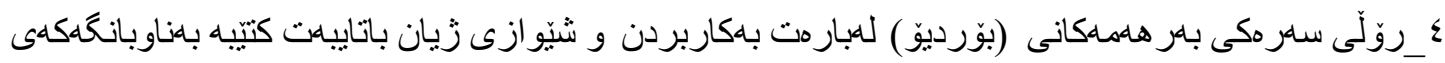

(جياوازى)(Reimer,1995)

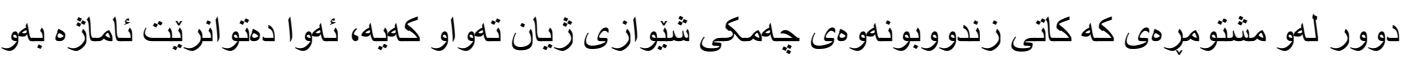

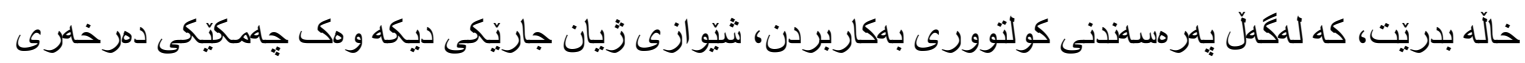

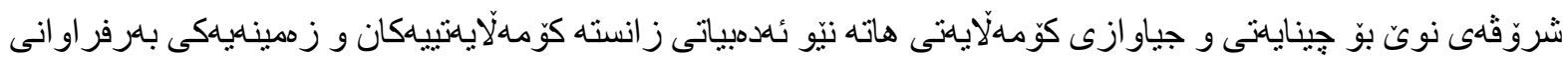

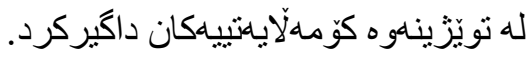

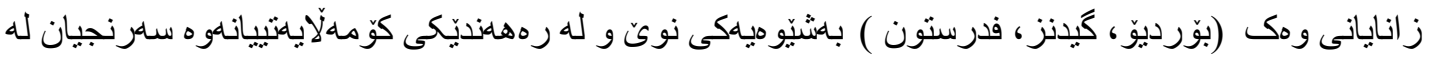

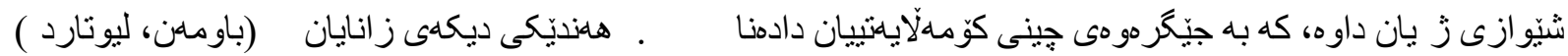

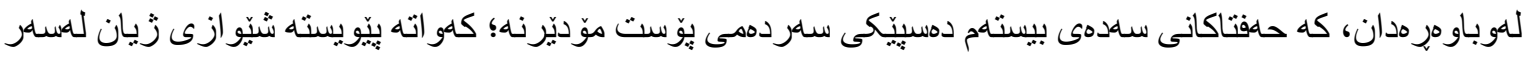

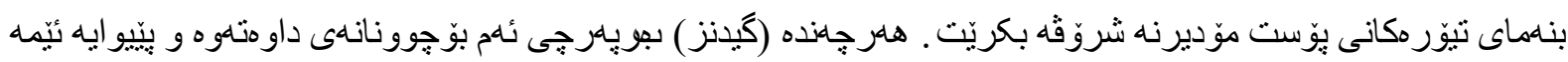

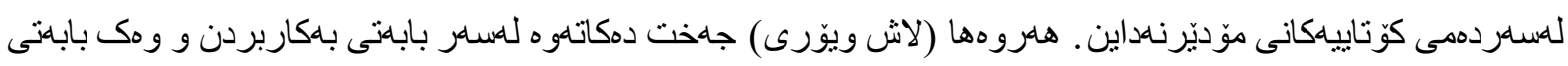

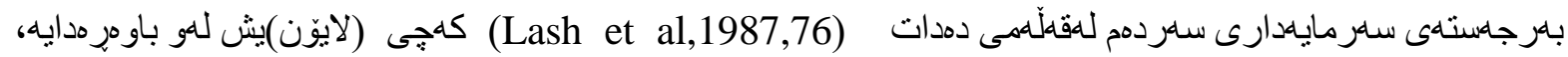

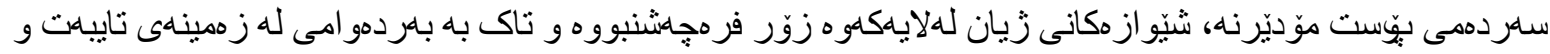

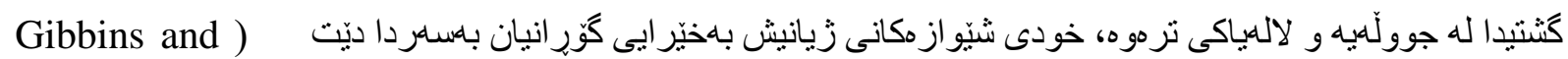

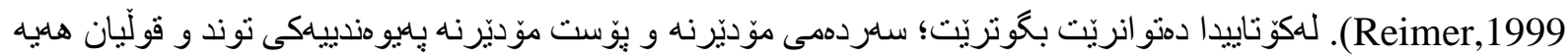

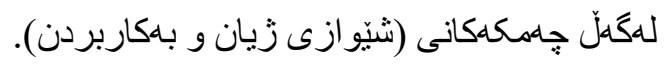

دووهم: جهمكى شيّوازى زيان

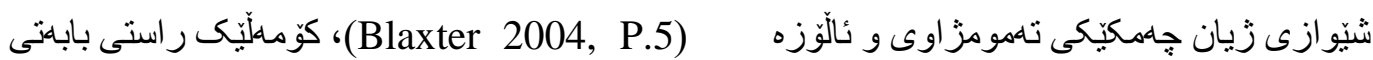

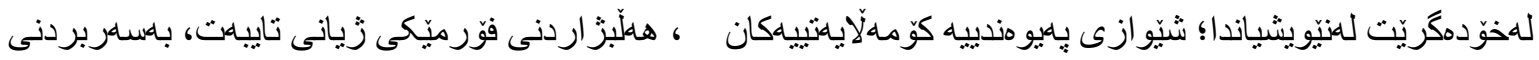

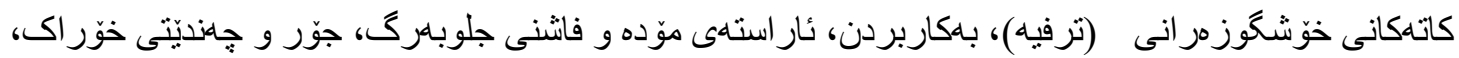




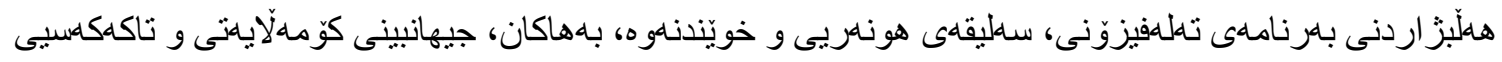

Bocock, and Thompson, 1992;Evanse and Jachson 2007; Featherstone 1991 )

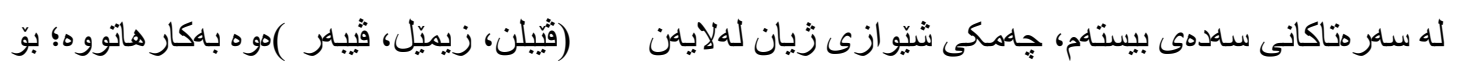

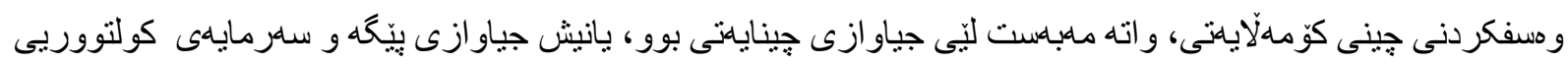

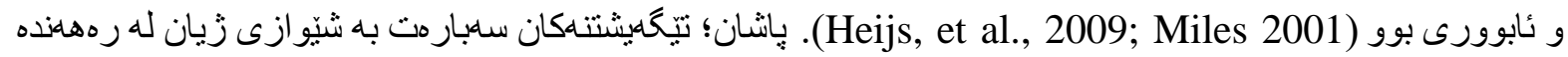

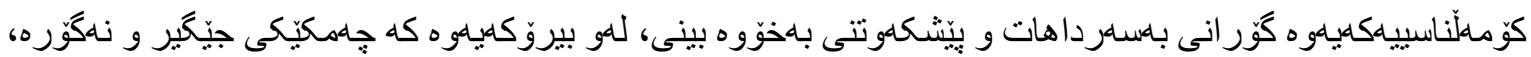

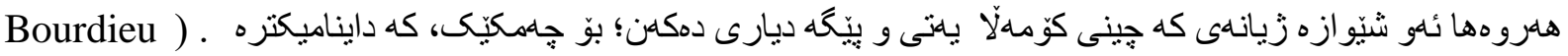
دهات 2007

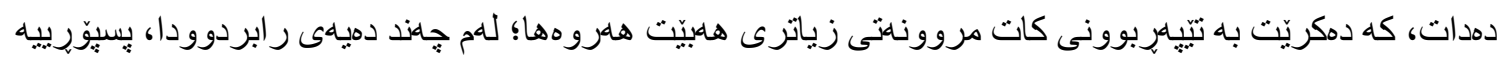

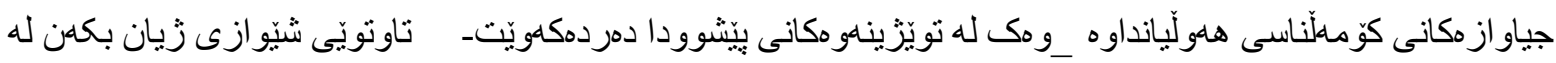

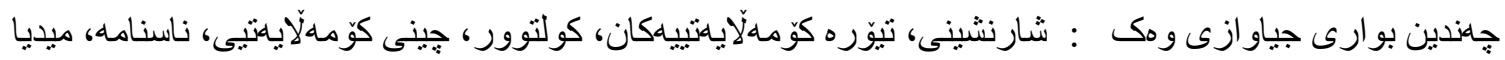

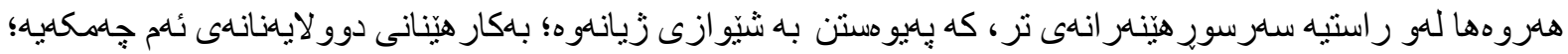

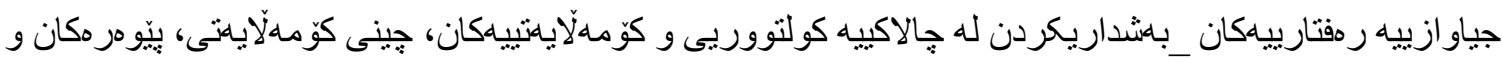
بهاكان. (Filipcová. and Tokarski, 1989.)

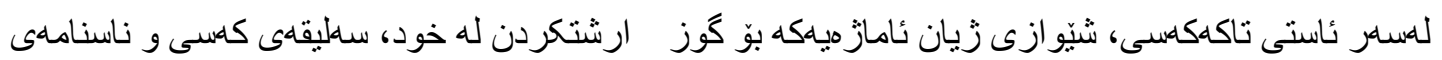

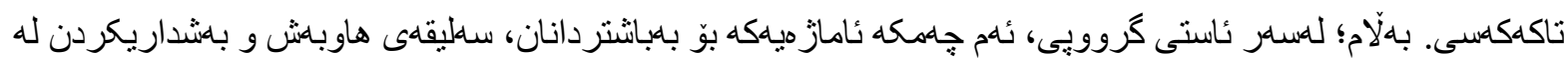

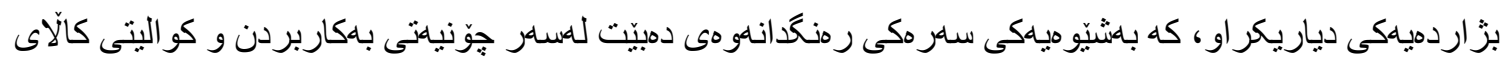

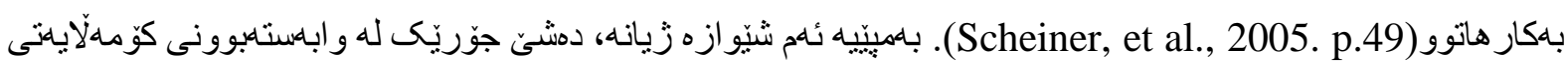

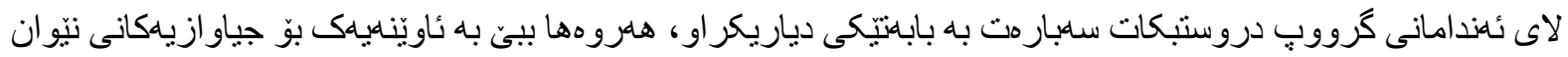

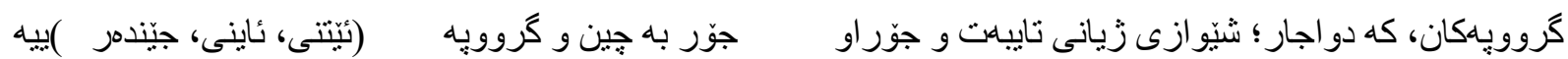

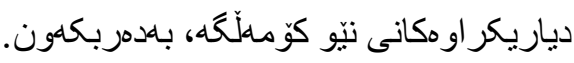

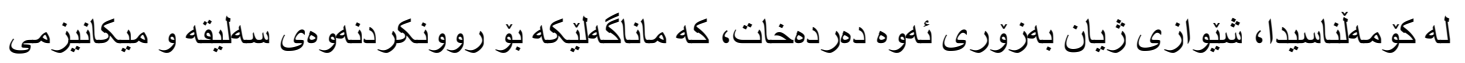

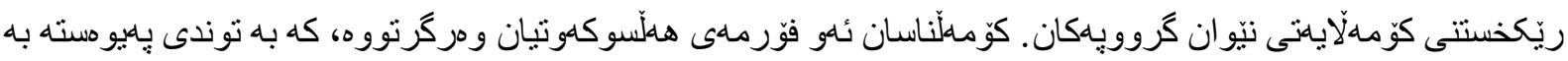
باككاربردناعوه (Mohammadpur and Mahmoodi, 2016; Wilska, 2002; Miles 2001).

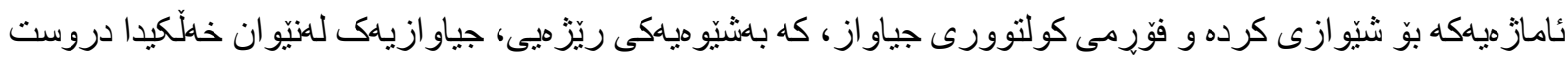
دمكات. Turner, 2006) ( T).

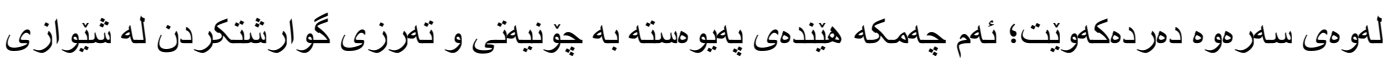

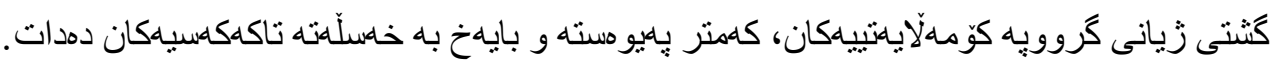

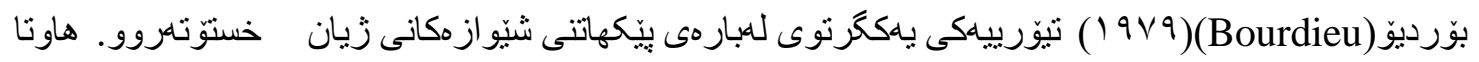

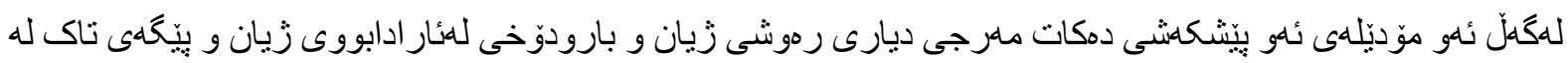

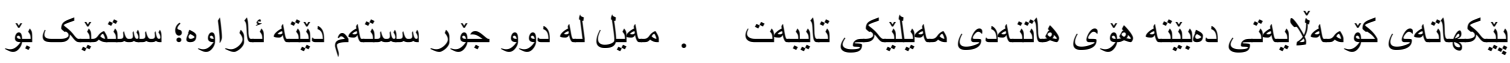

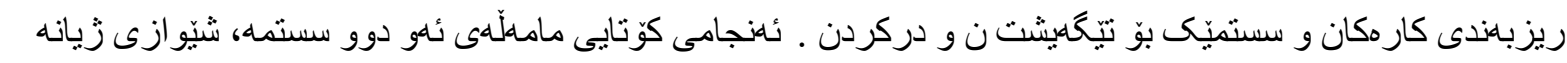

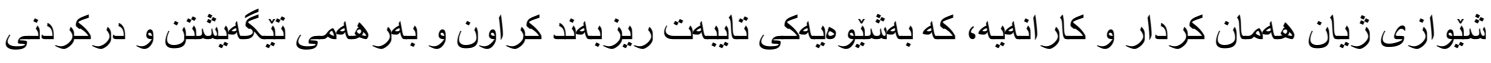

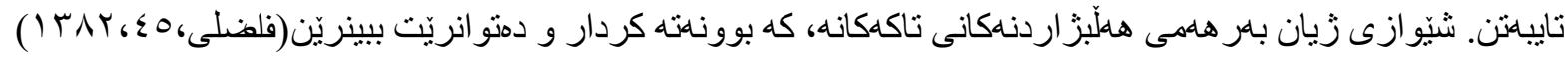




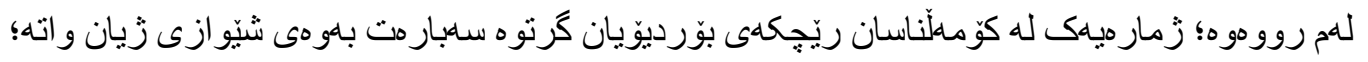

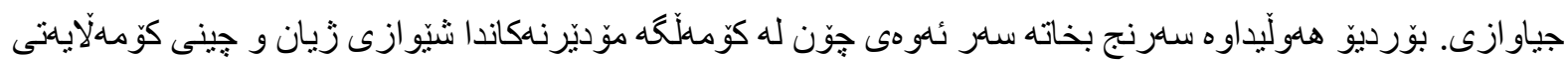

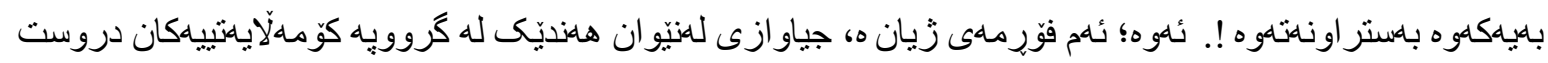

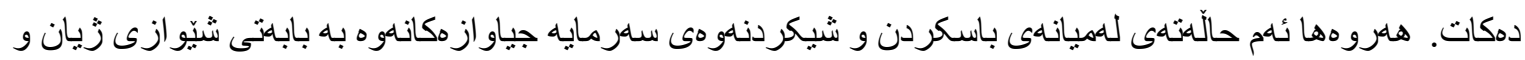

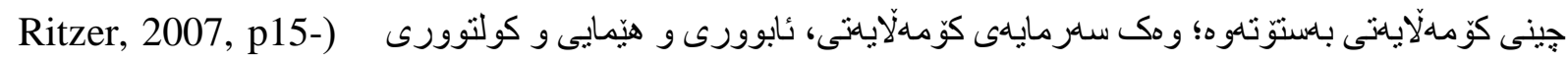

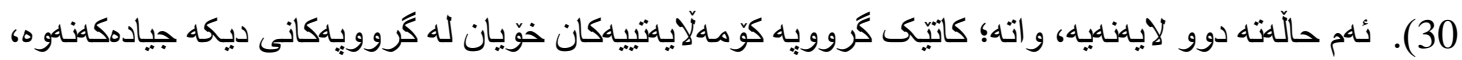

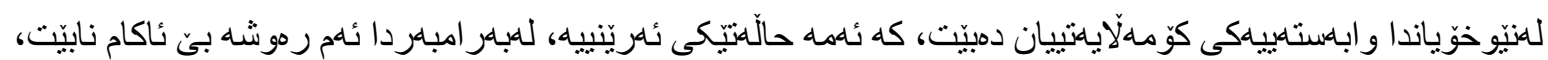

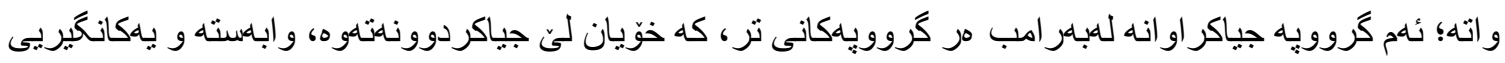

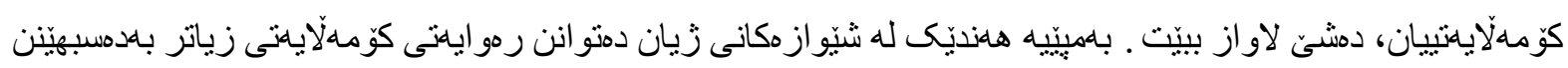

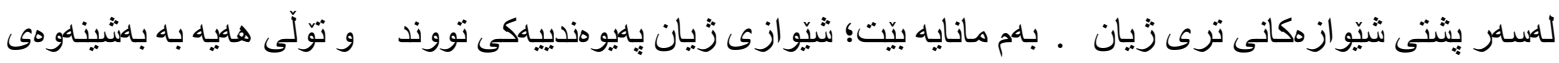

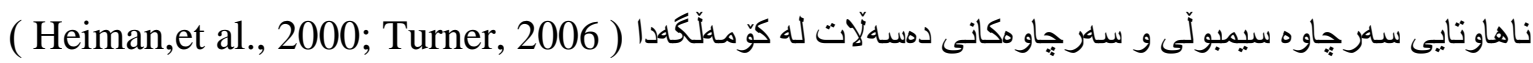

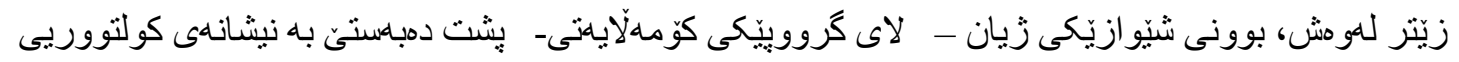

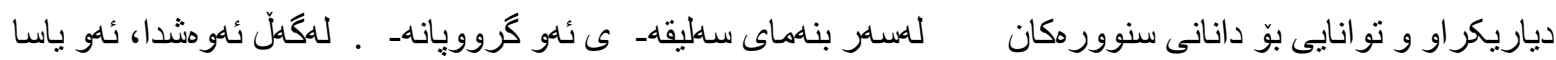

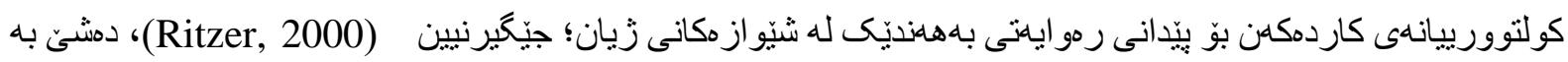

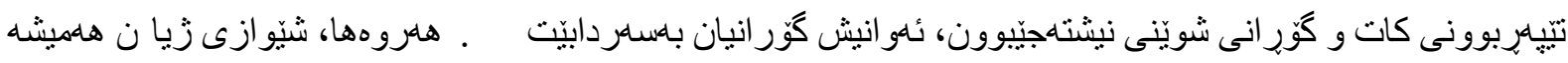

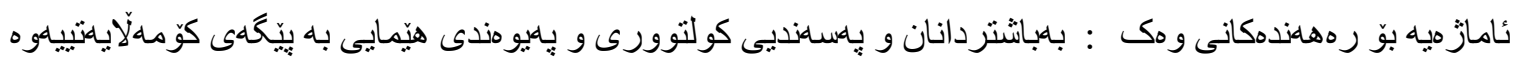

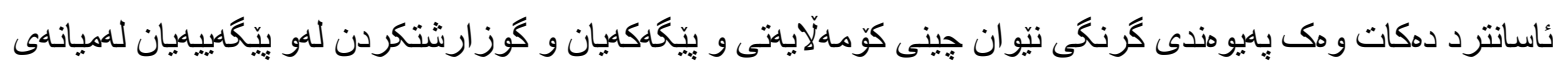

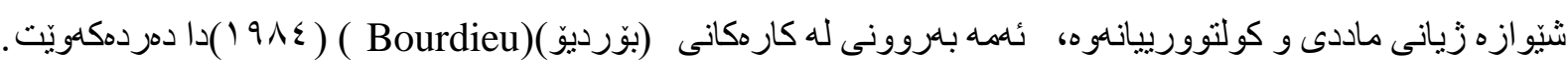

.(Bell, 2016)

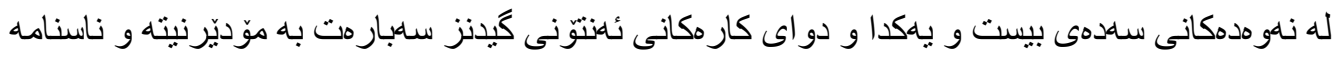

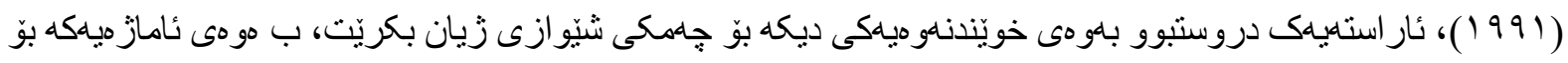

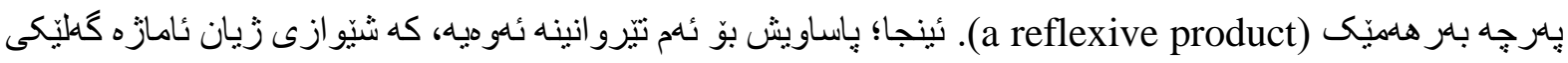

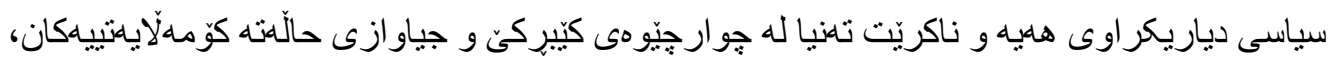

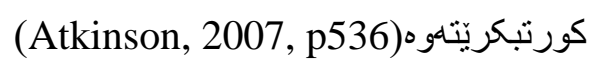

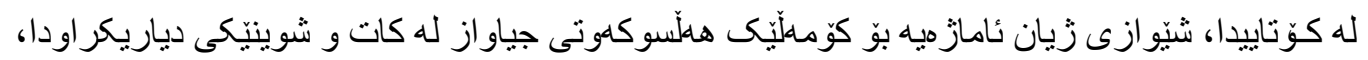

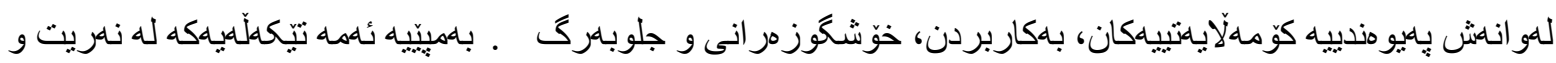

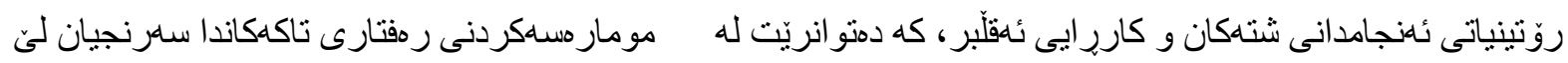

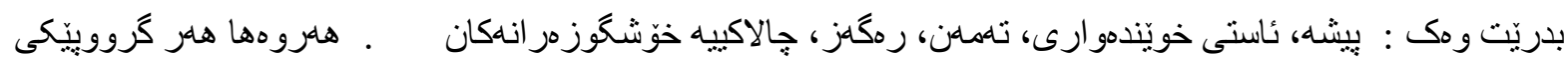

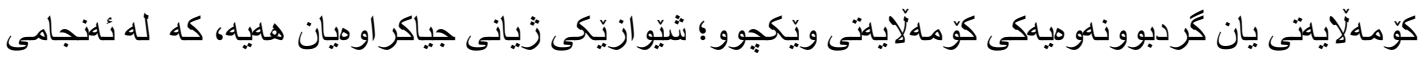

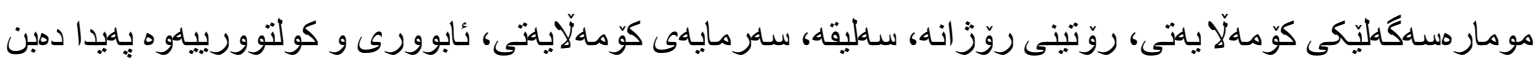

.(Bourdieu, 1979,1984,1985)

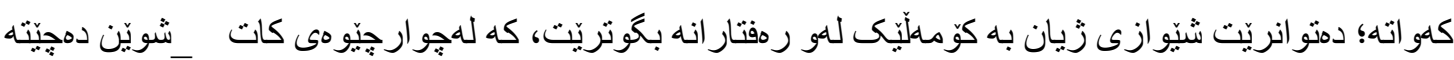

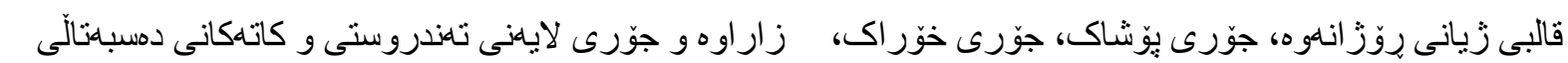

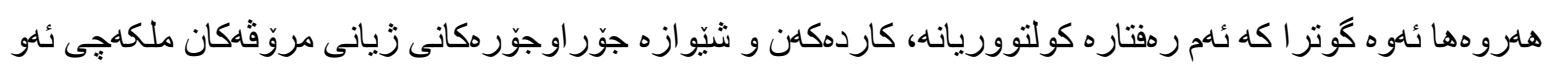




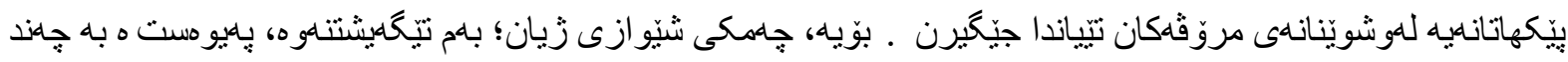

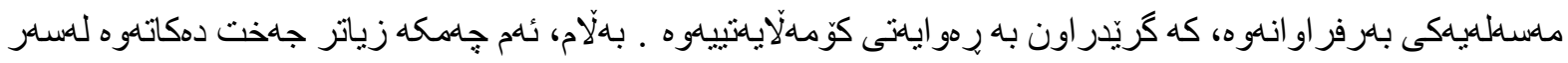

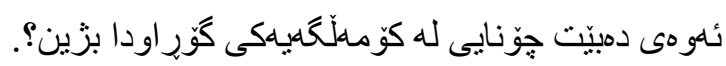

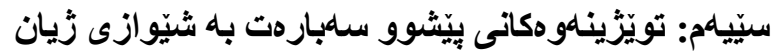

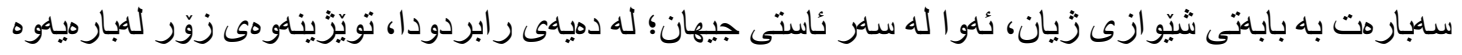

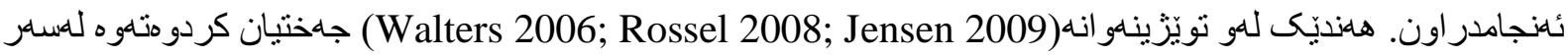

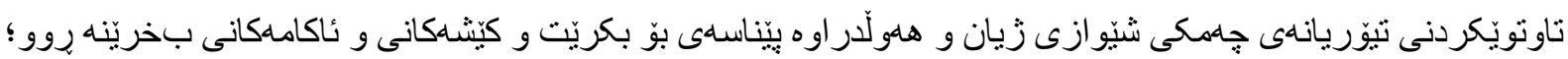

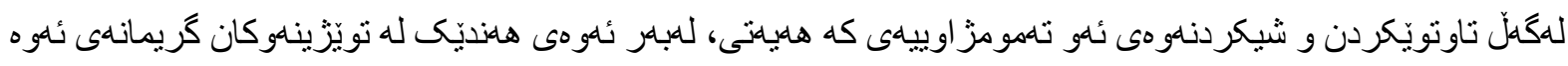

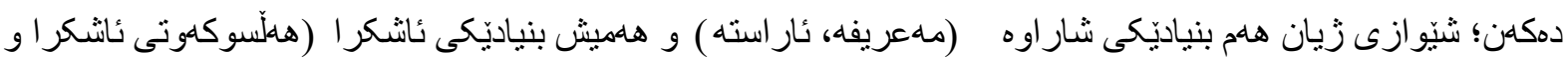

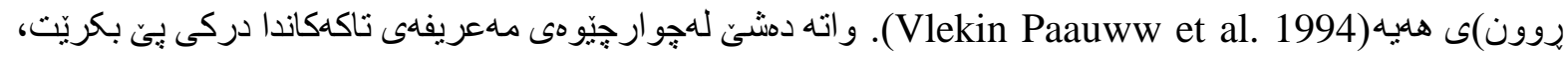

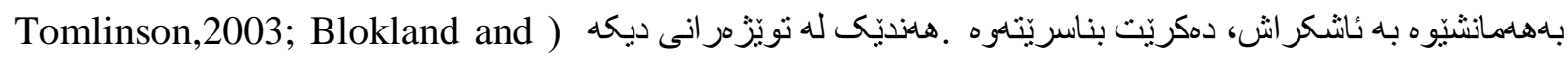

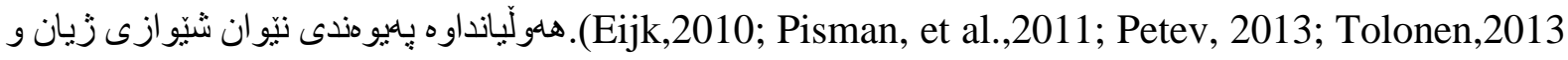

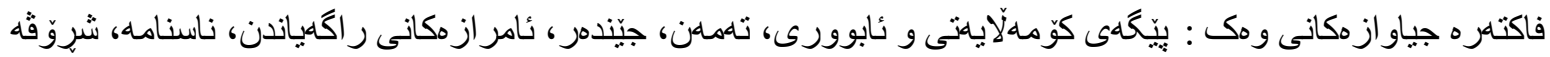

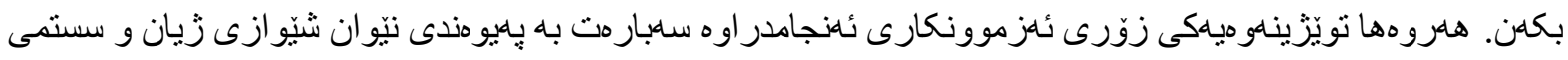

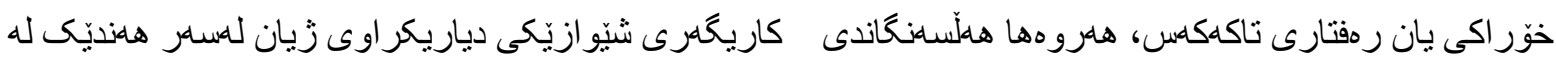
بابهتهكانى وهك: نهخوّشى و تهندروستى ( Donoghoe et al. 1992; Milligan et al. 1997; Heiman et al. 2000; Galobardes et al. 2003; Estaji et al. 2006; Tol et al. 2011; Pakholok 2013; Chan and .(Leung 2014

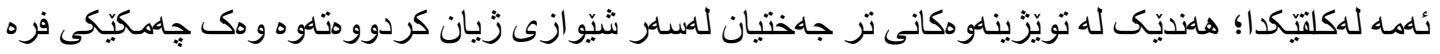

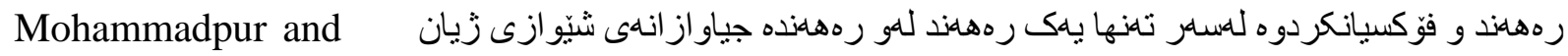
Mahmoodi, 2016; Wilska, 2002

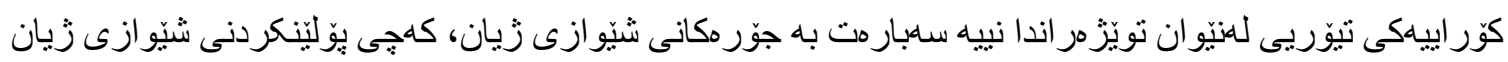

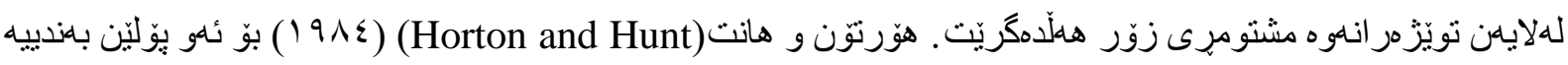

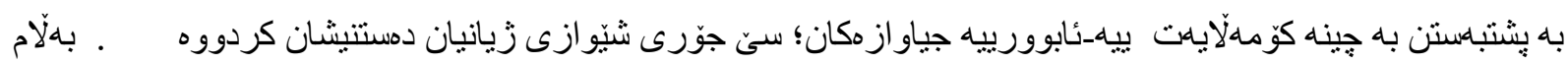

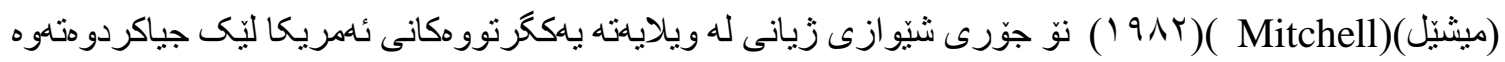

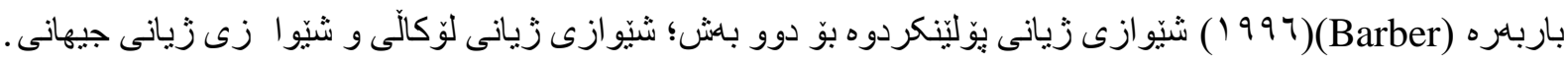

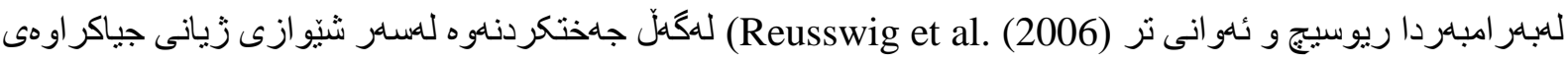

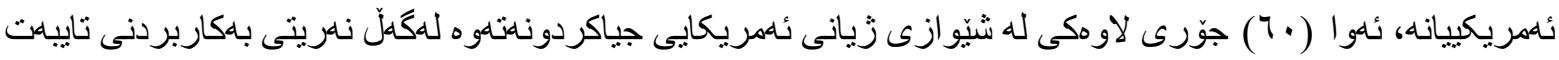

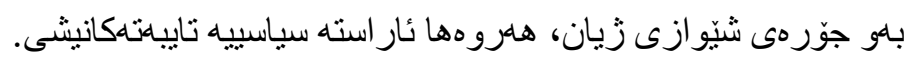

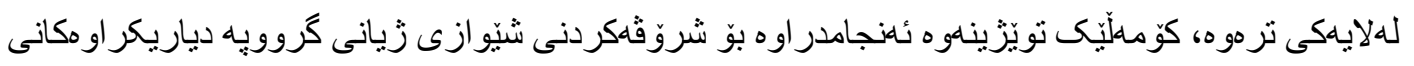

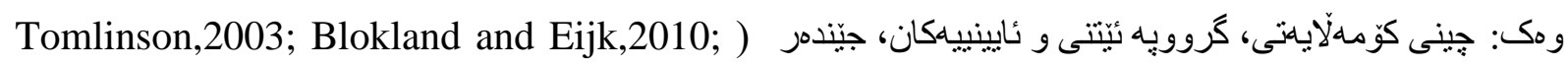
(SSY,2006; Diepen and Musterd, 2009; ) هلروهها، هلر ياك له (Pisman, et al.,2011; Petev, 2013 (Ge and Hokao, 2005; Cao, Maume and Jr,1993; Popkin, 1999; Fleischer, 2007 


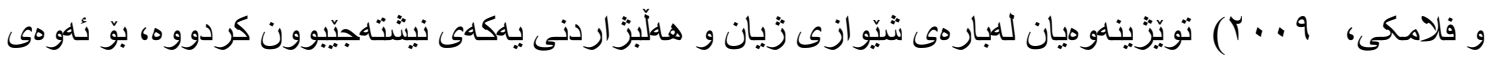

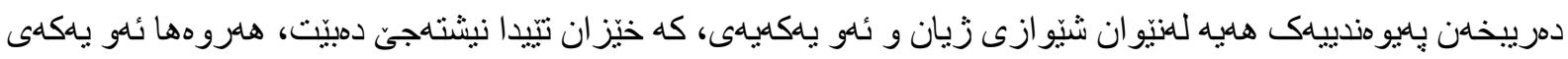

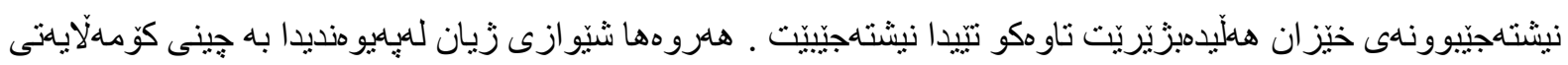

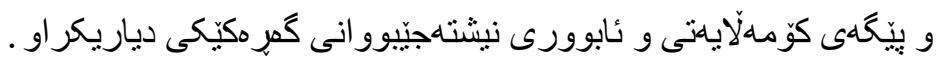

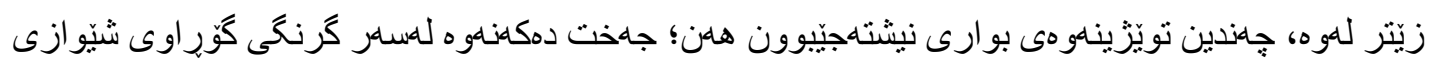

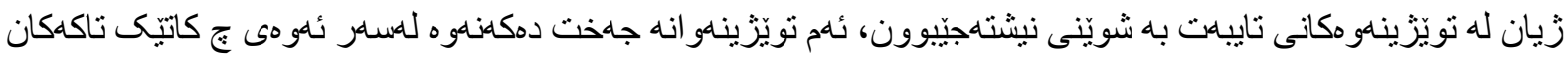

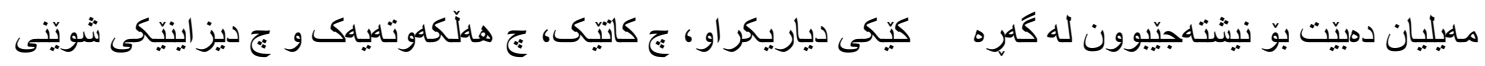

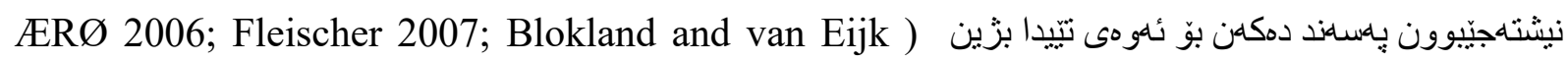

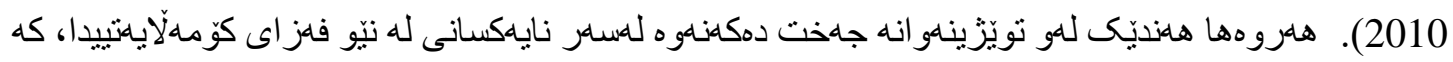

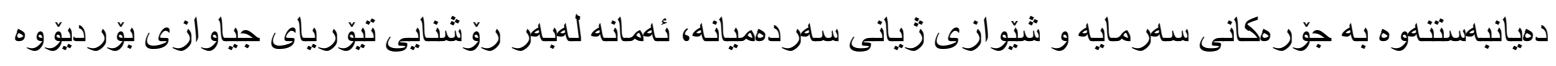

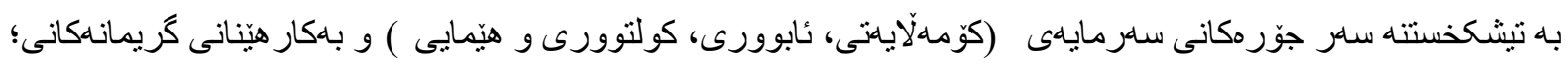

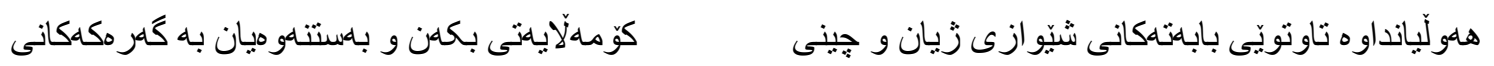

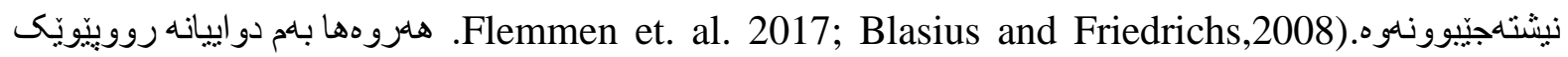

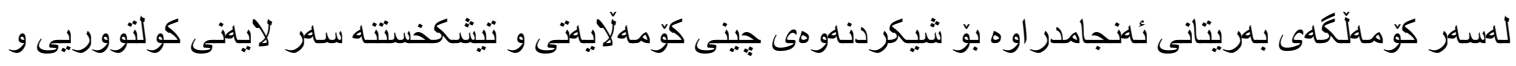

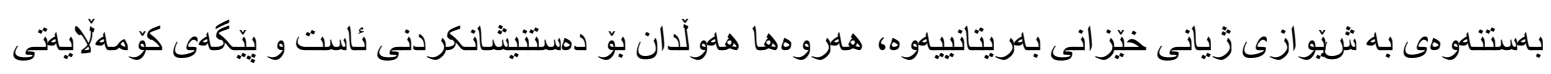

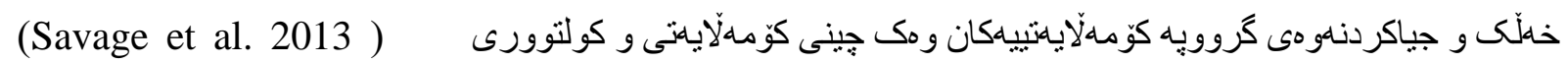

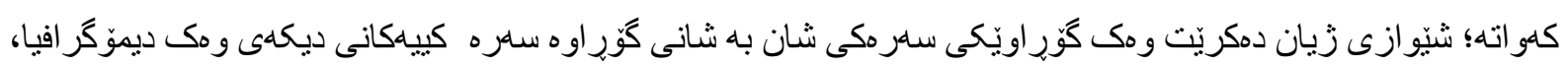

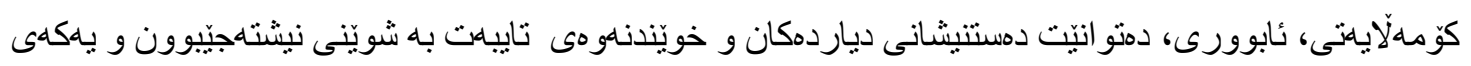

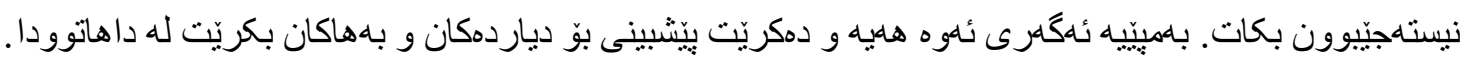

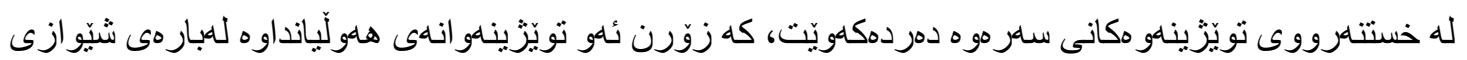

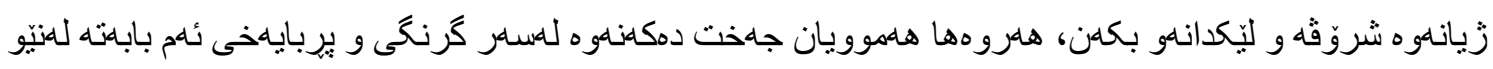

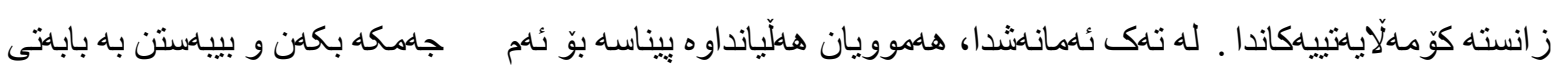

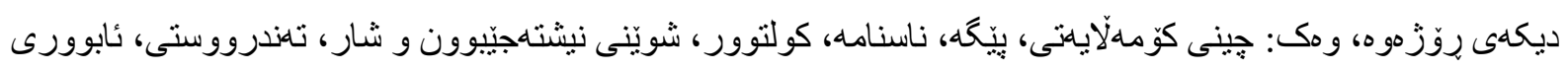
و باككاربردن.

\section{جوارهم: يارادايمهل تيوّرييهكانى شيّوازى زيان}

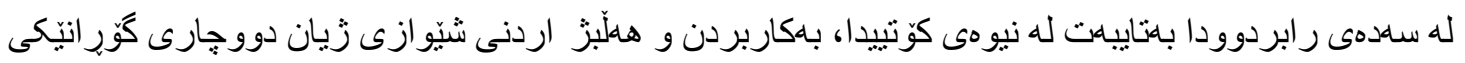

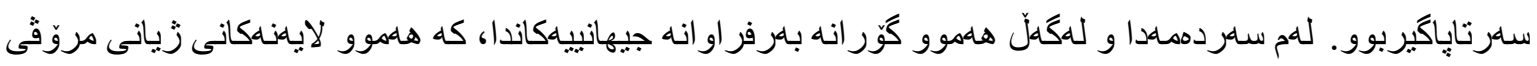

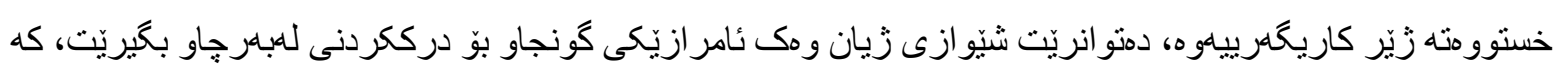

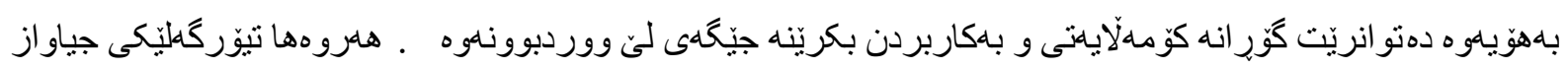

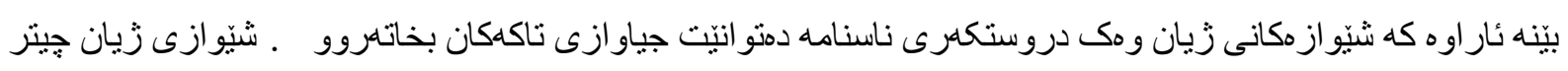

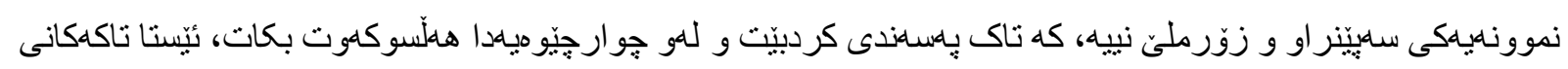

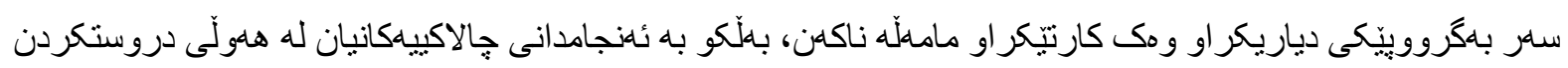

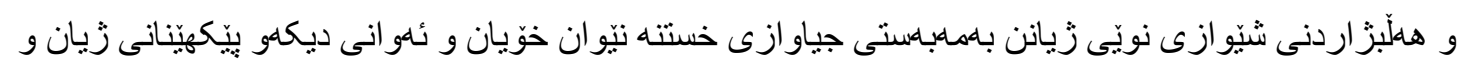




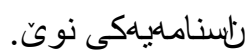

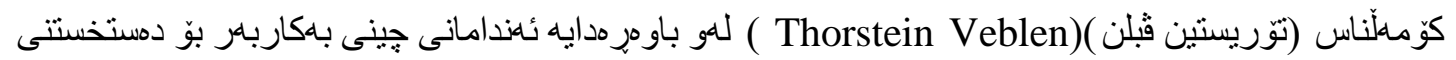

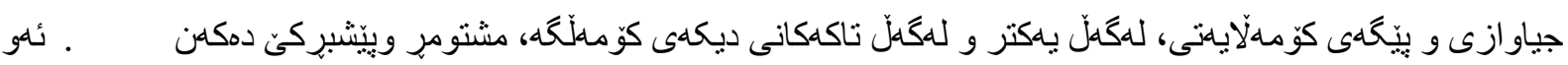

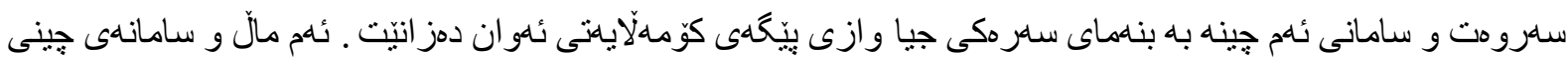

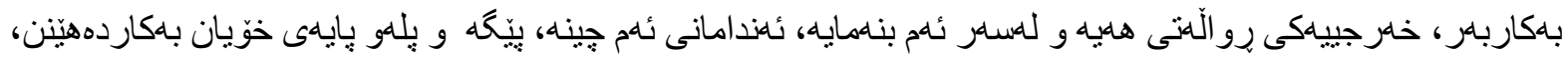

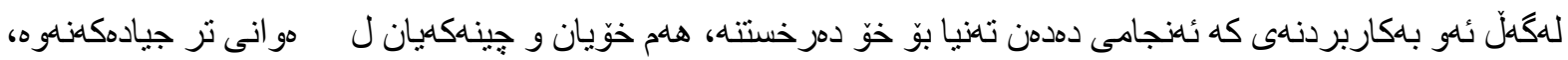

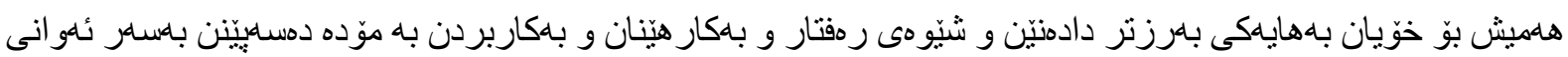

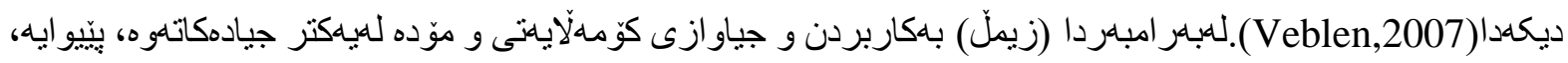

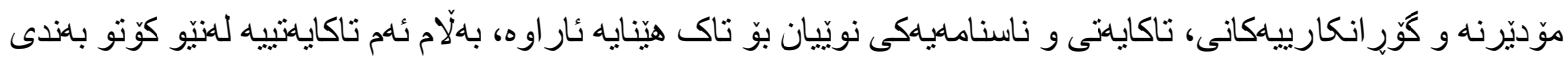

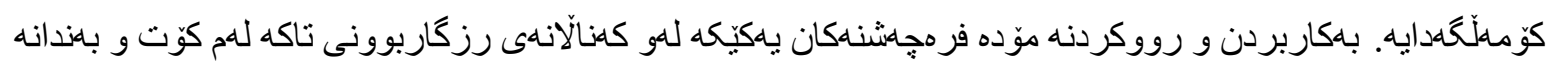

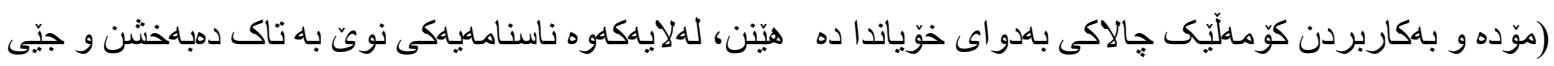

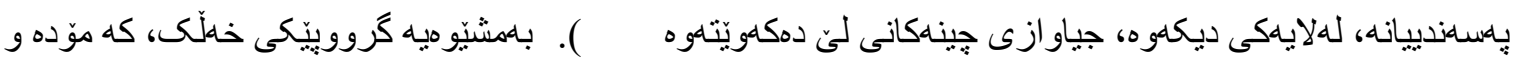

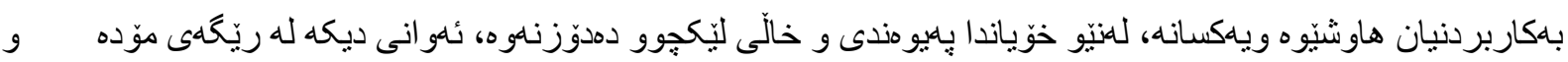

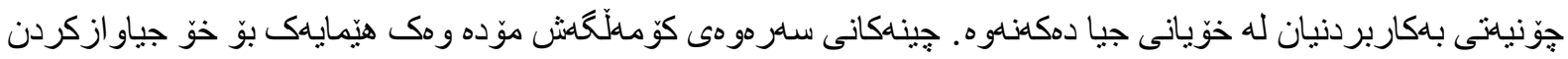

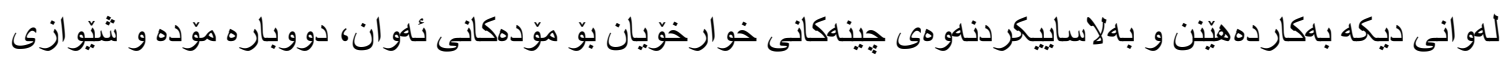

بهاربردنى نوى ديّننه ئار اوه (Clemens, 2000,

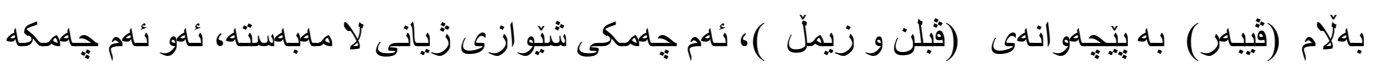

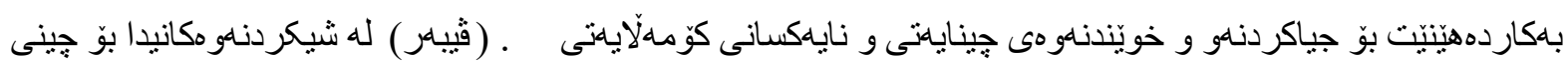

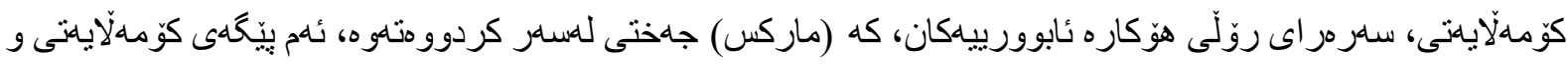

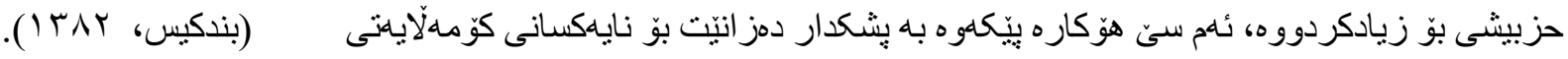

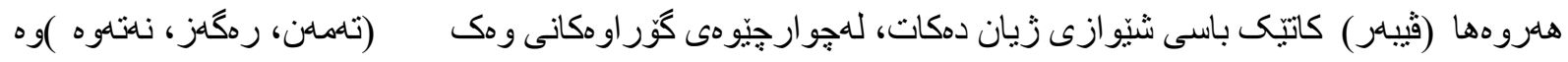

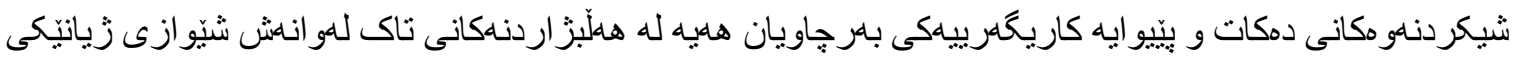

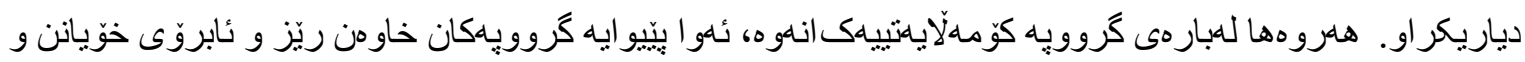

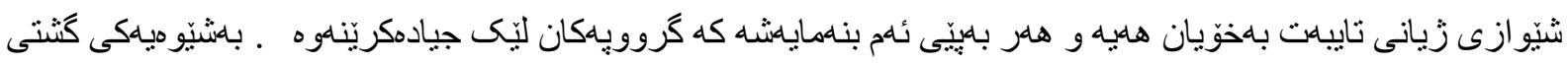

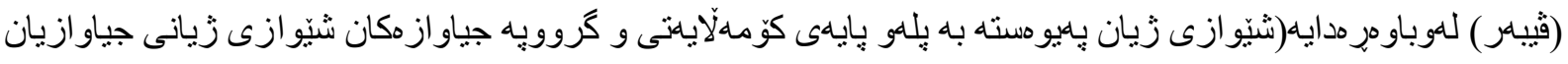

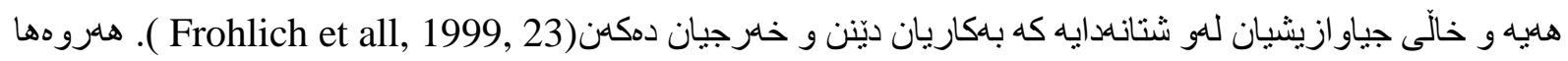

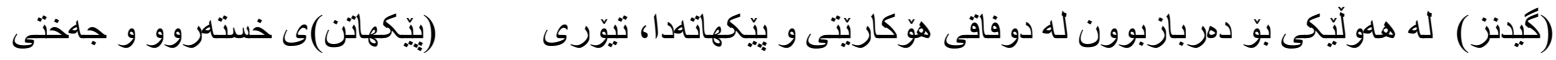

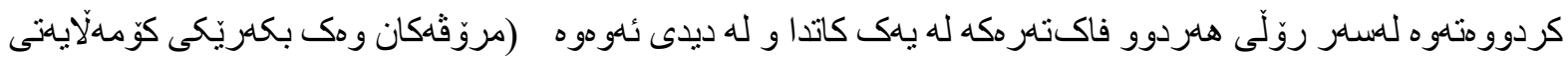

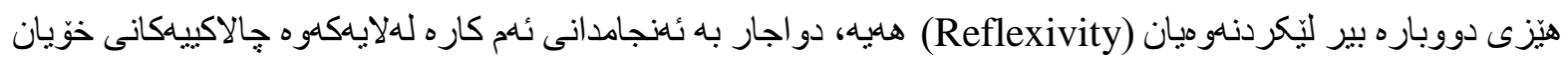

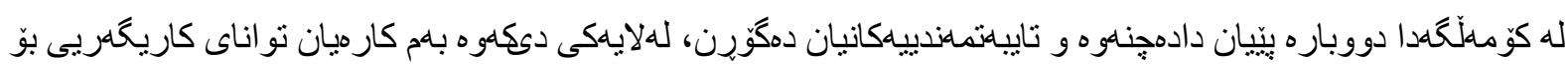

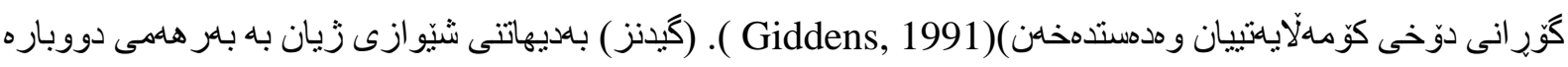

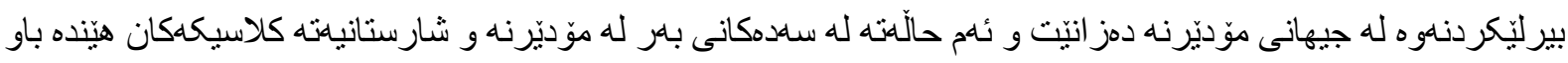

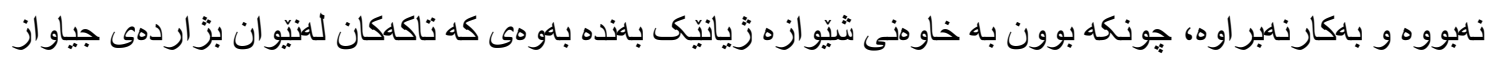

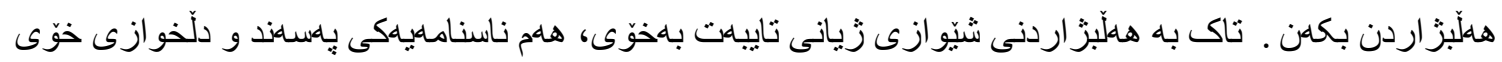




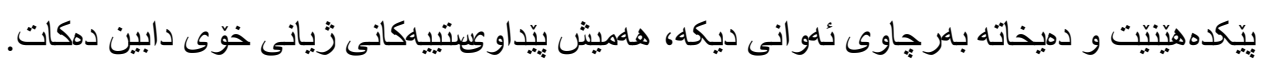

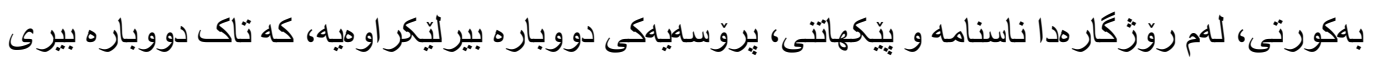

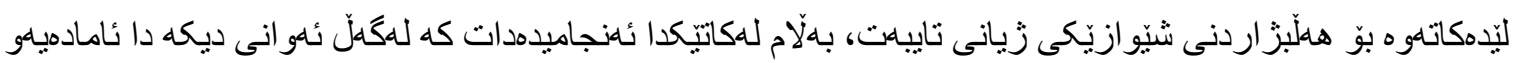

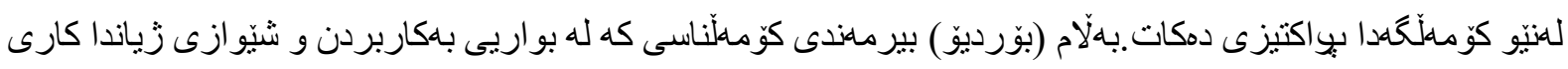

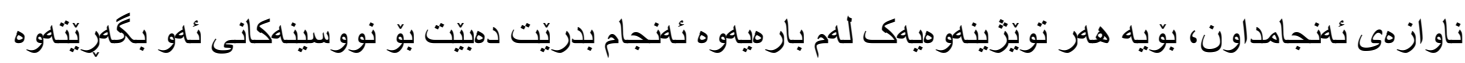

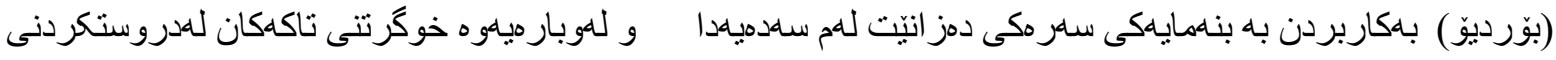

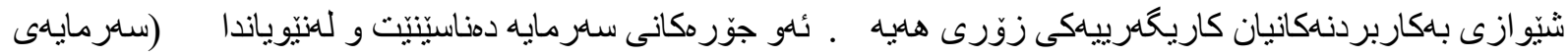

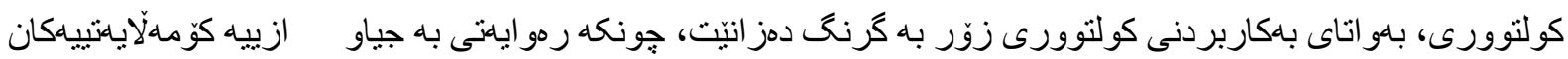

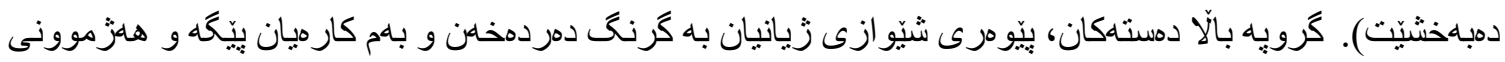

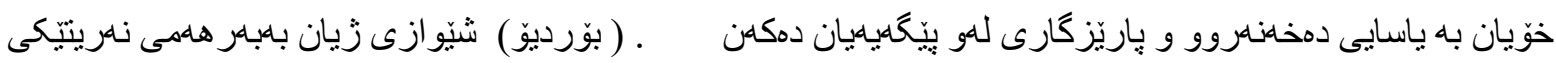

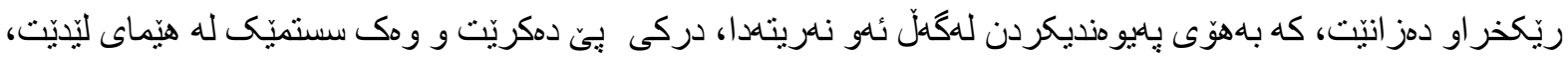

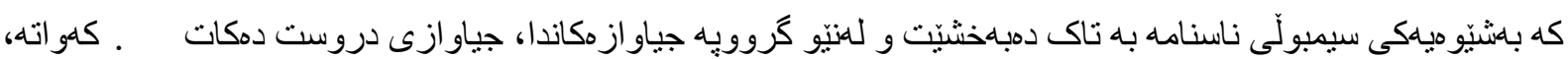

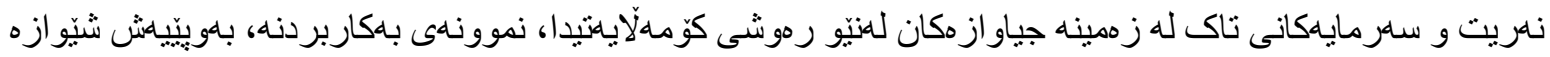

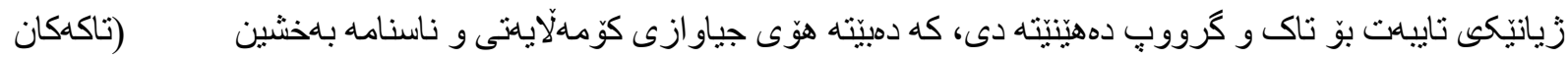

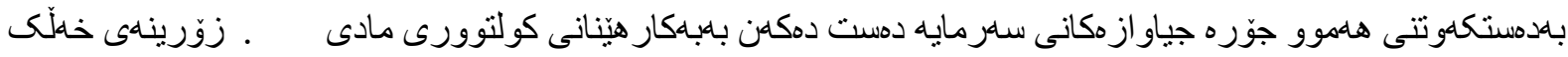

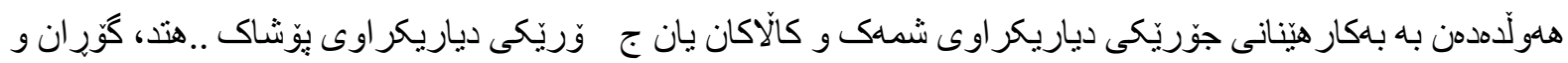

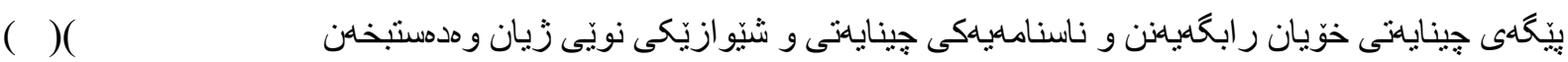

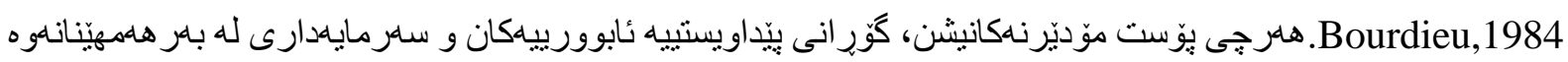

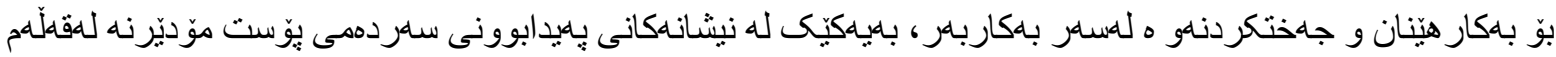

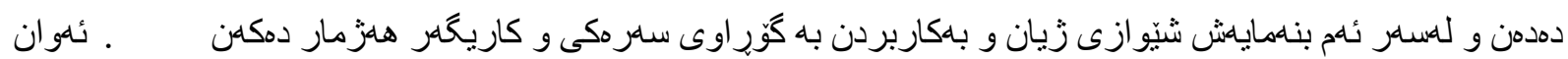

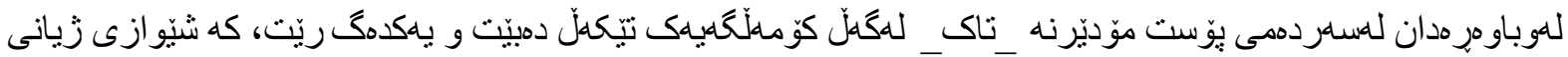

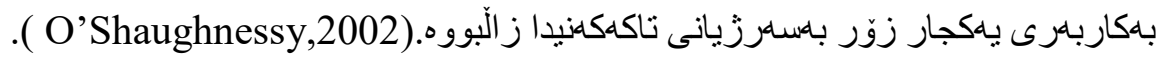

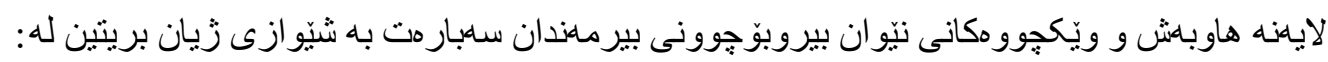

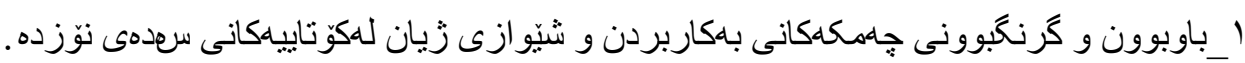

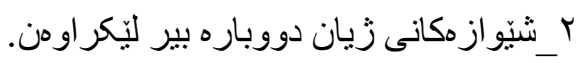

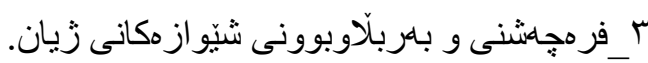

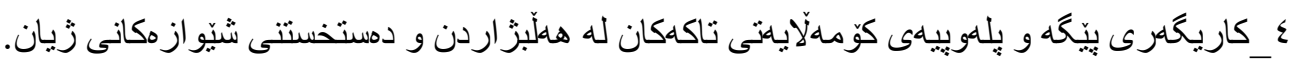

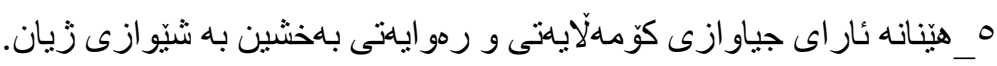

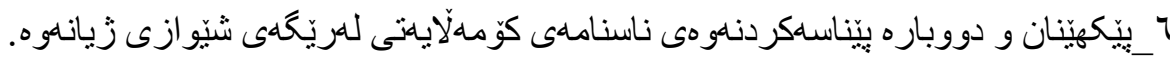

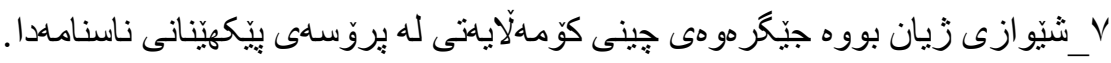

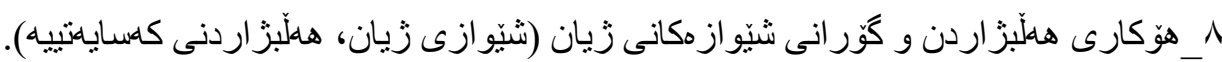

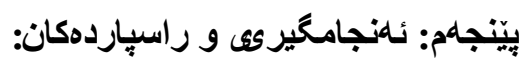

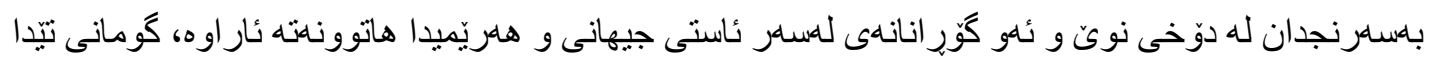




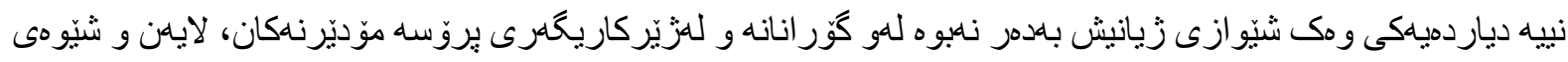

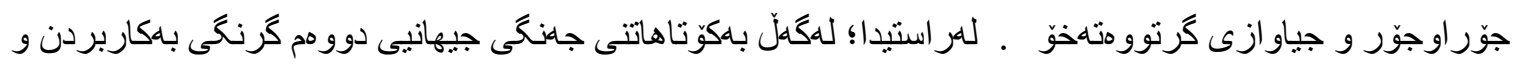

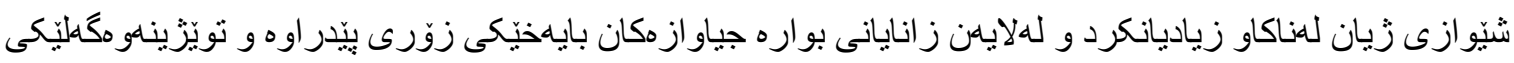

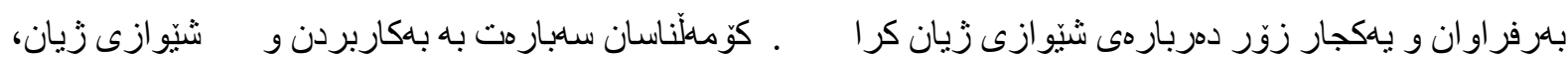

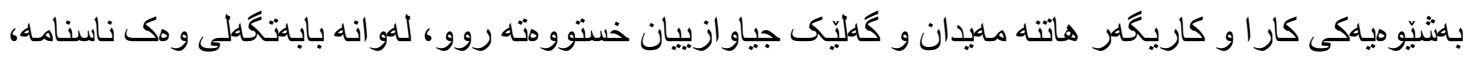

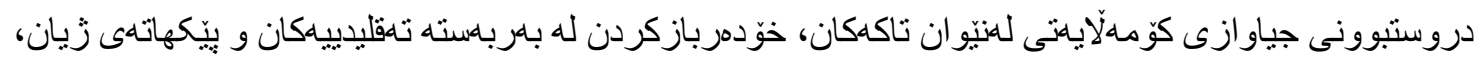

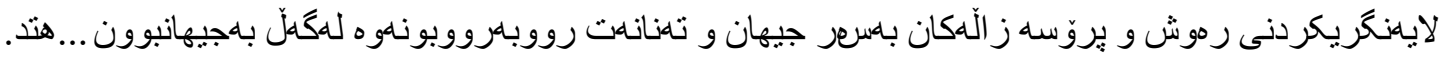

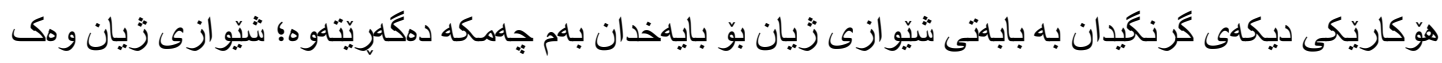

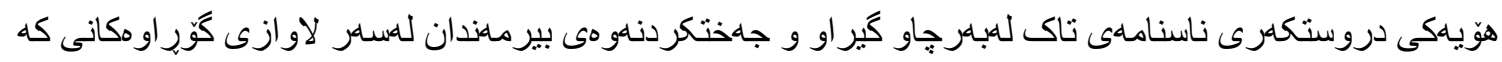

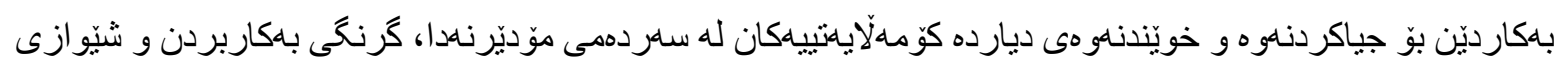

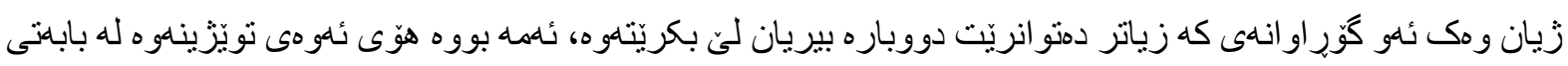

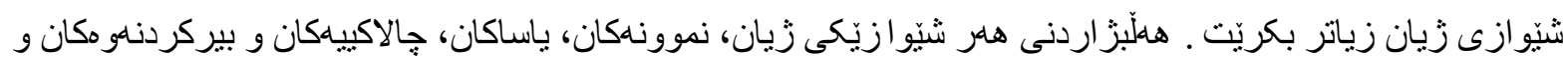

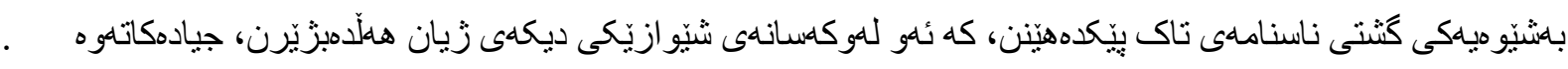

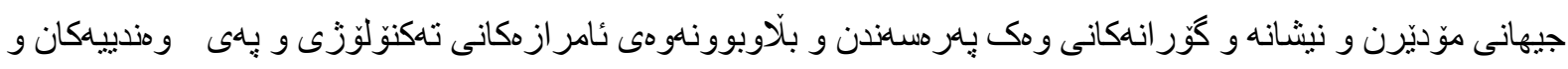

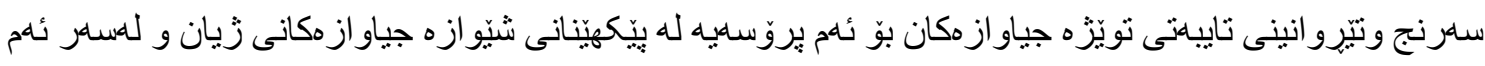

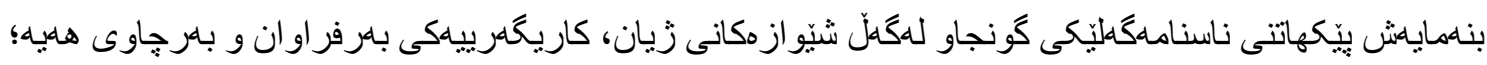

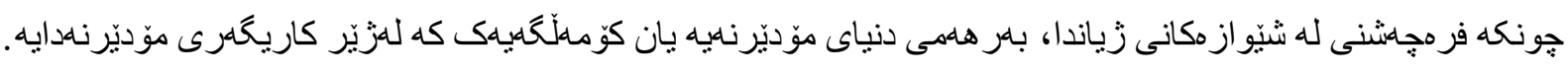

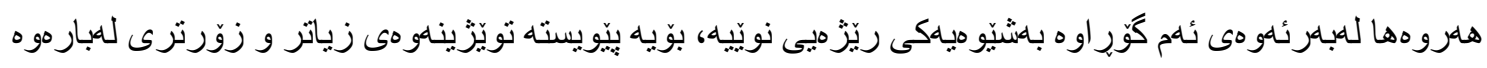

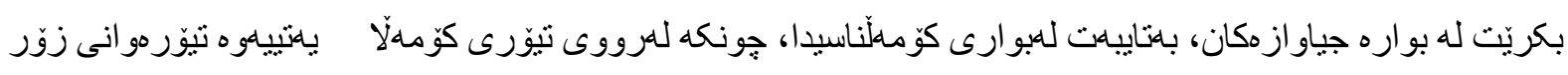

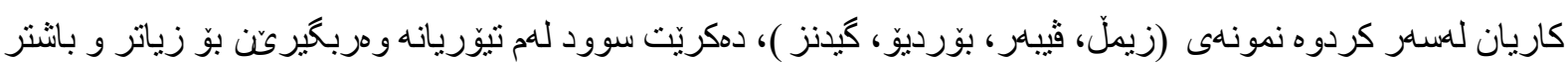

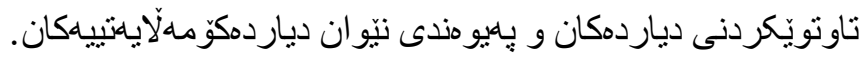




\section{References}

Arslan, Z. (2011). Urban Middle Class, Lifestyle and Taste in Keçiören and Çankaya, Ankara: Distinction through Home Furniture and, Furnishing and Decoration. Basılmamıs doktora tezi). Orta Doğu Teknik Üniversitesi/Sosyal Bilimler Enstitüsü, Ankara.

Atkinson, W.(2007). Anthony Giddens as adversary of class analysis. Sociology, 41(3), pp.533-549.

Barber, B.R.(1995). Jihad versus McWorld: How globalism and tribalism are reshaping the world. Tarmans Bokk: New York.

Baudrillard, J.(2016). The consumer society: Myths and structures. Sage.

Bell, W., 1958. Social choice, life styles, and suburban residence. The suburban community, 234.

Bendix, Reinhard.(2000).Max Weber: an Intellectual Portrait.Translation to Persian: Mahmud Ramyd, tahran: her ms.

Blasius, J. and Friedrichs, J.(2008). Lifestyles in distressed neighborhoods: A test of Bourdieu's "taste of necessity" hypothesis. Poetics, 36(1), pp.24-44.

Blaxter, M.,(2003). Health and lifestyles. Routledge.

Blokland, T. and Van Eijk, G. (2010). Do people who like diversity practice diversity in neighbourhood life? Neighbourhood use and the social networks of 'diversity-seekers' in a mixed neighbourhood in the Netherlands. Journal of Ethnic and Migration Studies, 36(2), pp.313-332.

Bocock, R. (2008). Consumption. Routledge.

Bocock, R. and Thompson, K. eds.( 1992). Social and cultural forms of modernity. Cambridge: Polity press.

Bögenhold, D.(2001). Social inequality and the sociology of life style: material and cultural aspects of social stratification. American Journal of Economics and Sociology, 60(4), pp.829-847

Bootsma, H.G.(1995). The influence of a work-oriented life style on residential location choice of couples. Journal of Housing and the Built Environment, 10(1), pp.45-63.

Bourdieu, P. (1984). Distinction: A social critique of the judgement of taste. Harvard university press.

Bourdieu, P. (1998). Practical reason: On the theory of action. Stanford University Press.

Chan, C.W. and Leung, S.F., (2015). Lifestyle health behaviors of Hong Kong Chinese: results of a cluster analysis. Asia Pacific Journal of Public Health, 27(3), pp.293-302.

Chawshyan, Hassan.(1996). Lifestyle and Social Identity.Tahran: Neshrny.

Evans, D. and Jackson, T.D.,( 2007). Towards a sociology of sustainable lifestyles. RESOLVE Working Paper Series, 3.

Fazly, Muhamad.(1997).Counsamption and Lifstyle,Tahran: qwm.

Featherstone, M. (1995). Undoing culture: Globalization, postmodernism and identity (Vol. 39). Sage.

Filipcová, B., Glyptis, S. and Tokarski, W. eds.(1989). Life styles: Theories, concepts, methods and results of life style research in international perspective. Institute for Philosophy and Sociology of the Czechoslovak Academy of Sciences.

Galobardes, B., Costanza, M.C., Bernstein, M.S., Delhumeau, C. and Morabia, A.,( 2003). Trends in risk factors for lifestyle-related diseases by socioeconomic position in 
Geneva, Switzerland, 1993-2000: health inequalities persist. American journal of public health, 93(8), pp.1302-1309.

Gibbins, J.R. and Reimer, B., (1999). The politics of postmodernity: An introduction to contemporary politics and culture. Sage.

Giddens, A.(1991). Modernity and self-identity: Self and society in the late modern age. Stanford university press.

Hall, A.(2004). Local and global, globalization and ethnicity (B. Barkat, Trans.). Organon: Philosophical, Literary and Cultural Quarterly, 24, pp.239-262.

Heijs, W., Carton, M., Smeets, J. and Van Gemert, A.(2009). The labyrinth of lifestyles. Journal of Housing and the Built Environment, 24(3), pp.347-356.

Heiman, A., Just, D.R. and Zilberman, D.(2000). The role of socioeconomic factors and lifestyle variables in attitude and the demand for genetically modified foods. Journal of Agribusiness, 18(3), pp.249-260.

Horton, P.B., Hunt, C.L.(1984): Sociology. McGraw-Hill Higher Education, New York

Jensen, M.( 2007). Defining lifestyle. Environmental sciences, 4(2), pp.63-73.

Jensen, M.( 2009). Lifestyle: suggesting mechanisms and a definition from a cognitive science perspective. Environment, development and sustainability, 11(1), pp.215-228.

Miles, S.,( 2001). Social theory in the real world. Sage.

Milligan, R.A., Burke, V., Dunbar, D.L., Spencer, M., Balde, E., Beilin, L.J. and Gracey, M.P.(1997). Associations between lifestyle and cardiovascular risk factors in 18-yearold Australians. Journal of Adolescent Health, 21(3), pp.186-195.

Mitchell, A.(1983). The nine American lifestyles: Who we are and where we're going. Scribner Book Company.

Mohammadpur, A. and Mahmoodi, K.(2016). Lifestyle and identity in contemporary Iranian Kurdistan (a grounded study of Marivan City). Quality \& Quantity, 50(5), pp.19071928.

Pisman, A.( 2007). Lifestyles as Centrifugal and Centripetal Forces in the Polycentric Network City of Flanders. In International Conference on New Concepts and Approaches for Urban and Regional Policy and Planning, Leuven 2-3/4/2007 papers.

Ritzer, G.(2000). Modern sociological theory, 5th edition. McGraw-Hill

Rössel, J.(2007). Conditions for the explanatory power of life styles. European Sociological Review, 24(2), pp.231-241.

Scheiner, J. and Kasper, B.(2005). A lifestyles approach to investigating residential mobility and travel behaviour. Spatial planning, urban form and sustainable transport,pp.4260.

Shasty, Shimaand Flamky, Mohamad.(2009).Relations btween Lifestyle and Housing in Iran, Journal of Social Saences, Vol.6, NO.3.

Southerton, D.(2001). Consuming kitchens: taste, context and identity formation. Journal of Consumer Culture, 1(2), pp.179-203.

Steven Seidman.(2004).The struggle of Sociology.Translation to Persian: Hady Jalil, Tahtan: Harms.

Stevenson, N.(2002). Understanding media cultures: Social theory and mass communication. Sage.

Swartz, D.(2012). Culture and power: The sociology of Pierre Bourdieu. University of Chicago Press.

Tolonen, T.(2013). Youth cultures, lifestyles and social class in Finnish contexts. Young, 21(1), pp.55-75.

Tomlinson, M. (2003). Lifestyle and social class. European Sociological Review, 19(1), pp.97-111.

Turner, B.S. (2006). The Cambridge Dictionary of Sociology. Cambridge university press. 
Veblen, T.(2007). Theory of the Leisure Class (Oxford world's classics).Transaction Publishers.

Walters, G.D.( 2006). Lifestyle theory: Past, present, and future. Nova Publishers. 